Application of An optimized anNotation pipeline to the CRYPtococcus DEUTEROgatTII GENOME REVEALS DYNAMIC PRIMARY METABOLIC GENE CLUSTERS AND GENOMIC IMPACT OF RNAI LOSS

Patrícia Aline GRÖHS FERRAREZE ${ }^{*, \ddagger}$ Corinne MAUFRAIS ${ }^{*}{ }^{\dagger}$, Rodrigo SILVA ARAUJO STREIT ${ }^{\ddagger}$, Shelby J. PRIEST $^{\S}$, Christina CUOMO**, Joseph HEITMAN ${ }^{\S}$, Charley Christian STAATS ${ }^{\ddagger, 2}$, Guilhem JANBON*,1,2

* Institut Pasteur, Unité Biologie des ARN des Pathogènes Fongiques, Département de Mycologie, F-75015, Paris, France

† Institut Pasteur, HUB Bioinformatique et Biostatistique, C3BI, USR 3756 IP CNRS, F-75015, Paris, France

¥ Programa de Pós-Graduação em Biologia Celular e Molecular, Centro de Biotecnologia, Universidade Federal do Rio Grande do Sul, Porto Alegre, Brazil.

$\S$ Department of Molecular Genetics and Microbiology, Duke University Medical Center, Durham, NC, 27710, USA

** Broad Institute of MIT and Harvard, Cambridge, MA 02142

1 Corresponding author

2. Both authors should be considered as senior authors 
Running Tittle: $C$. deuterogattii genome annotation

Key words: Cryptococus deuterogattii; Genome annotation pipeline; RNAi; Metabolic gene

Address of the corresponding author: cluster

\section{Guilhem Janbon}

Institut Pasteur, Unité Biologie des ARN des Pathogènes Fongiques, Département de Mycologie, 25 rue du Dr Roux,75015, Paris, France 
40

41

\section{Abstract}

Evaluating the quality of a de novo annotation of a complex fungal genome based on RNA-seq data remains a challenge. In this study, we sequentially optimized a Cufflinks-CodingQuary based bioinformatics pipeline fed with RNA-seq data using the manually annotated model pathogenic yeasts Cryptococcus neoformans and Cryptococcus deneoformans as test cases. Our results demonstrate that the quality of the annotation is sensitive to the quantity of RNA-seq data used and that the best quality is obtained with 5 to 10 million reads per RNA-seq replicate. We also demonstrated that the number of introns predicted is an excellent $a$ priori indicator of the quality of the final de novo annotation. We then used this pipeline to annotate the genome of the RNAideficient species Cryptococcus deuterogattii strain R265 using RNA-seq data. Dynamic transcriptome analysis revealed that intron retention is more prominent in $C$. deuterogattii than in the other RNAi-proficient species C. neoformans and C. deneoformans. In contrast, we observed that antisense transcription was not higher in C. deuterogattii than in the two other Cryptococcus species. Comparative gene content analysis identified 21 clusters enriched in transcription factors and transporters that have been lost. Interestingly, analysis of the subtelomeric regions in these three annotated species identified a similar gene enrichment, reminiscent of the structure of primary metabolic clusters. Our data suggest that there is active exchange between subtelomeric regions, and that other chromosomal regions might participate in adaptive diversification of Cryptococcus metabolite assimilation potential. 
bioRxiv preprint doi: https://doi org/10.1101/2020.09.01 278374 this version posted December 14,2020 . The copyright holder for this preprint (which was not certified by peer review) is the author/funder, who has granted bioRxiv a license to display the preprint in perpetuity. It is made available under aCC-BY-NC-ND 4.0 International license.

\section{Introduction}

In recent years, we have seen an astonishing multiplication of fungal genome sequences

(JAMES et al. 2020). Long-read sequencing and adapted bioinformatics tools are quickly improving as well. It is expected that telomere-to-telomere whole-genome sequencing will soon become standard for reference genomes of diverse organisms (GIORDANo et al. 2017; DAL Molin et al. 2018; YADAv et al. 2018). Yet, fungal genomes remain difficult to annotate. Historically, most annotation tools have relied upon comparative genomics, but other pipelines utilize RNA-seq data or a combination of both approaches to propose gene annotation models (CANTAREL et al. 2008; HAAS et al. 2011; Min et al. 2017; HARIDAS et al. 2018). These pipelines are very efficient in intron-poor species, at least for predicting coding regions. For instance, a recent MAKER-based optimized pipeline tested on 39 budding yeast genomes missed only $3.9 \%$ of genes and $4.8 \%$ of exons, on average (SHEN et al. 2018). However, the results were poorer in intron-rich species, for which gene annotation is challenging. Even when RNA-seq data are available, it is still very difficult to correctly predict the exon-intron structure primarily because fungal exons can be extremely short (JANBON et al. 2014), but also because these genomes are compact. Thus, when tested on fungal data sets, de novo transcriptome assemblers like Trinity (GRABHERR et al. 2011) or Cufflinks (TRAPNELL et al. 2010) tend to predict very large transcripts with no biological relevance. Nevertheless, several pipelines have been published and sequencing centers like the Joint Genome Institute (JGI) and the Broad Institute have developed specialized pipelines to produce annotation drafts, which are very useful in large-scale comparison analyses (HAAS et al. 2011; HARIDAS et al. 2018). Some methods, like the construction of large deletion collections, or precise analysis of gene content needs more precise annotation, and the annotation strategy applied will depend on the goal of the research (MUDGE AND HARROW 2016). Manual curation of a pre-annotated genome will likely result in the highest-quality gene prediction. Some tools, like Artemis (CARVER et al. 2012) and Apollo (DunN et al. 2019), have been used to manually curate annotation, but they are time 
consuming even when several annotators are implemented. Without manual curation, it is impossible to anticipate the results from an annotation bioinformatics pipeline fed with RNA-seq data. Typically, the quality of the prediction will depend on the diversity, quantity, and quality of the data, but no a priori indicator exists to determine if the de novo gene prediction is accurate. Pathogenic Cryptococcus species are basidiomycete yeasts, which cause nearly 200,000 deaths annually around the world (KWON-CHUNG et al. 2014). There are currently eight recognized pathogenic species of Cryptococcus (HAGEN et al. 2015; Farrer et al., 2019). Manual annotation of the Cryptococcus neoformans and Cryptococcus deneoformans reference genomes revealed complex and dynamic transcriptomes (JANBON et al. 2014; WALLACE et al. 2020). These annotations were recently completed through precise identification of the transcript leader (TL) and 3'UTR sequences through TSS-seq and 3UTR-seq analyses; these annotations are likely the most complete and detailed annotations in intron-rich fungi (WALLACE et al. 2020). With 99.5\% of 6,795 annotated coding genes containing introns, five to six introns per coding gene, and 37,832 introns in total, an automatic annotation of these genomes would be considered highly challenging even with the large sets of RNA-seq data that have been produced (WILM et al. 2007; JANBON 2018; WALLACE et al. 2020).

In this study, we compared the performances of three annotation pipelines fed with RNAseq data. We gradually optimized the quality of the de novo annotation using the well-annotated genomes of $C$. neoformans and $C$. deneoformans as ground-truth inputs. We found that the quantity of data used should not be too large and that the number of introns predicted had a positive, linear relationship with the quality of the de novo annotation. We used this pipeline to reannotate the reference genome of the RNAi-deficient Cryptococcus deuterogattii strain R265 using RNA-seq data. Analysis of the transcriptome dynamics of these three Cryptococcus species revealed that although the sense/antisense transcript ratio is similar across all three species, 
110 intron retention is higher in C. deuterogattii. Comparative gene content analysis identified a list of

111 genes that are absent or largely truncated in R265, many of which have been implicated in RNAi-

112 mediated silencing in Cryptococcus species. Finally, we also identified several primary metabolic

113 gene clusters (MGCs) that are absent in R265 and associated this loss with the subtelomeric gene

114 content. Our data suggest an active exchange of MGCs between subtelomeric regions and more

115 central regions of the genome. This exchange might contribute to the adaptive diversification of

116 metabolite assimilation potential in Cryptococcus.

MATERIALS AND METHODS

RNA-Seq sample and data production

RNA-seq libraries from four growth conditions (exponential phase at $30^{\circ} \mathrm{C}$, exponential phase at

$37^{\circ} \mathrm{C}$, stationary phase at $30^{\circ} \mathrm{C}$, and stationary phase at $\left.37^{\circ}\right)$, conducted in triplicate, of $\mathrm{C}$. neoformans $\mathrm{H} 99$ and $\mathrm{C}$. deneoformans JEC21 used in this study have been previously described (WALLACE et al. 2020). The C. deuterogattii R265 strain was grown in YPD at $30^{\circ} \mathrm{C}$ and $37^{\circ} \mathrm{C}$ under agitation to exponential or early stationary phase as previously described (WALLACE et al. 2020). Briefly, early stationary phase was obtained after $18 \mathrm{~h}$ of growth (final OD $600=15$ ) starting from at OD600 $=0.5$. Each Cryptococcus cell preparation was spiked in with one tenth (OD/OD) of $S$. cerevisiae strain FY834 cells grown in YPD at $30^{\circ} \mathrm{C}$ in stationary phase. Cells were washed, snap frozen and used to prepare RNA and total DNA samples. Biological triplicates were prepared in each 
134 DNA PCR-Free (Illumina). Then, 100 bases were sequenced from both ends using an Illumina

135 HiSeq2500 instrument according to the manufacturer's instructions (Illumina). For the mating 136 condition, total RNA was isolated (in biological triplicates) from a C. neoformans cross between the

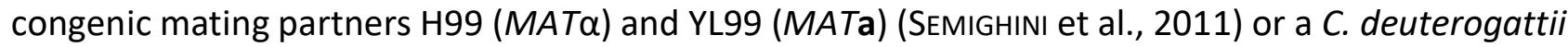
cross between the congenic mating partners R265 (MATa) and AIR265 (MATa) (ZHU et al. 2013). Briefly, overnight cultures were grown under standard laboratory conditions in YPD at $30^{\circ} \mathrm{C}$.

Overnight cultures were diluted to an $\mathrm{OD} 600=1.0$, and cells from both strains were mixed, spotted 141 onto $\mathrm{V} 8(\mathrm{pH}=5)$ mating medium, and incubated in the dark at room temperature for $48 \mathrm{~h}$. Cells were scraped from mating plates, snap frozen, and RNA was isolated using Trizol following the manufacturer's protocol. RNA quality was confirmed by Bioanalyzer (Agilent) and RNA samples were

to the manufacturer's instructions (Illumina).

\section{RNA-Seq library trimming and rRNA cleaning}

149 The paired reads from the RNA-seq libraries were trimmed for low quality reads and Illumina TruSeq 150 adapters were removed with Cutadapt v1.9.1 (MARTIN 2011) with the following parameters: --trim151 qualities 30 -e (maximum error rate) 0.1 --times 3 --overlap 6 --minimum-length 30 . The cleaning of 152 rRNA sequences was performed with Bowtie2 v2.3.3 (LANGMEAD AND SALZBERG 2012) with default 153 parameters; unmapped paired reads were reported using option --un-conc to identify reads that did 154 not align with rRNA sequences.

\section{RNA-Seq library mapping}

156 The cleaned reads from RNA-seq paired-end libraries from C. neoformans H99, C. deneoformans 157 JEC21, and C. deuterogattii R265 were mapped against their reference genomes (NCBI Genome 
Assemblies GCA_000149245.3, GCA_000091045.1 and GCA_002954075.1) with Tophat2 v2.0.14

159 (KIM et al. 2013) and the following parameters: minimum intron length 30; minimum intron

160 coverage 30; minimum intron segment 30; maximum intron length 4000; maximum multihits 1 ;

161 microexon search; and library-type fr-firststrand or fr-secondstrand (according to the RNA-seq

162 library).

163

164

165

166

167

168

169

170

\section{Pipeline selection}

The RNA-seq mapped reads from C. neoformans $\mathrm{H} 99$ and C. deneoformans JEC21 from the EXPO30 condition (exponential growth at $30 \mathrm{C}$ ) were tested in the three pipelines for gene prediction. BRAKER1 (HOFF et al. 2016) was performed with the default parameters plus the exclusion of alternative transcripts (--alternatives-from-evidence=false) using the three replicates $(A, B$, and $C)$ as RNA-seq source. Cuff-CQ (Cufflinks v2.1.1 ((TRAPNELL et al. 2010)) /Coding Quarry v2.0 (TeSTA et al. 2015)) and C3Q (Cufflinks v2.1.1/Cuffmerge/Coding Quarry v2.0) were tested with the basic parameters: minimum intron length (30); maximum intron length (4000); minimum isoform fraction (0.9); and overlap radius (10). The merged BAM file generated by the three replicates ( $A$, $B$, and $C)$ and used in the Cuff-CQ pipeline was obtained with Samtools merge. C3Q was performed separately for the three BAM files; the GTF files generated by the three predictions (for replicates $A, B$, and C) were then combined by Cuffmerge and the resulting transcripts were processed by CodingQuarry. The evaluation of the pipeline sensitivity and precision for gene prediction was performed by comparing the predicted annotations against the H99 and JEC21 reference annotations (WALLACE et al., 2020) with the GFFCompare program (PerTEA AND PeRTEA 2020).

For a better understanding, the C3Q pipeline with the basic Cufflinks parameters is named as C3Q1 protocol in the results section.

\section{Cufflinks parameters selection}


181 The selection of the best Cufflinks parameter combination was also performed with EXPO30 RNA-

182 seq libraries from $C$. neoformans $\mathrm{H} 99$ and $C$. deneoformans JEC21 according to the C3Q pipeline. For 183 this, the Cufflinks transcript assembly generated for each EXPO30 replicate (A, B, and C) was tested 184 with fixed and variable parameter combinations (Table 1). Subsequently, as established for the C3Q 185 pipeline, the predicted GTFs were merged and processed by CodingQuarry. All combinations include minimum intron length 30; maximum intron length 4000; and minimum isoform fraction 0.9; since we want to remove all isoforms. The variable parameters include: pre-mRNA fraction 0.15 to 1.0; 188 small anchor fraction 0.0; minimum fragments per transfag 1; overlap radius 1, 10 and 100; 3' 189 trimming (--trim-3-avgcov-thresh and -trim-3-dropoff-frac) 0 . The evaluation of the Cufflinks 190 parameters for sensitivity and specificity for gene prediction was performed by comparison of the 191 predicted annotations against the H99 and JEC21 reference annotations with the GFFCompare 192 program.

193 For a better understanding, the C3Q pipeline with the " $Q$ " Cufflinks parameters (selected 194 combination) is named as C3Q2 protocol in the results section. 
Table 1.

\section{Cufflinks parameter combinations}

A $\quad$--max-intron-length 4000 --min-intron-length 30 --min-isoform-fraction 0.9

B $\mid$--max-intron-length 4000 --min-intron-length 30 --min-isoform-fraction 0.9 --min-frags-pertransfag 1

C --max-intron-length 4000 --min-intron-length 30 --min-isoform-fraction 0.9 --pre-mrna-fraction 0.25

D $\mid$--max-intron-length 4000 --min-intron-length 30 --min-isoform-fraction 0.9 --overlap-radius 10

E --max-intron-length 4000 --min-intron-length 30 --min-isoform-fraction 0.9 --overlap-radius 100

F $\mid$--max-intron-length 4000 --min-intron-length 30 --min-isoform-fraction 0.9 --trim-3-avgcovthres 0 --trim-3-dropoff-frac 0.0

G $\quad$--max-intron-length 4000 --min-intron-length 30 --min-isoform-fraction 0.9 --pre-mrna-fraction 0.25 --overlap-radius 10 --trim-3-avgcov-thresh 0 --trim-3-dropoff-frac 0.0

H $\mid$--max-intron-length 4000 --min-intron-length 30 --min-isoform-fraction 0.9 --pre-mrna-fraction 0.25 --overlap-radius 10 --trim-3-avgcov-thresh 0 --trim-3-dropoff-frac 0.0 --min-frags-pertransfag 1

I $\mid$--max-intron-length 4000 --min-intron-length 30 --min-isoform-fraction 0.9 --pre-mrna-fraction 0.25 --overlap-radius 1 --trim-3-avgcov-thresh 0 --trim-3-dropoff-frac 0.0

J --max-intron-length 4000 --min-intron-length 30 --min-isoform-fraction 0.9 --pre-mrna-fraction 0.25 --overlap-radius 10 --trim-3-avgcov-thresh 0 --trim-3-dropoff-frac 0.0 --min-frags-pertransfag 1

K $\mid$--max-intron-length 4000 --min-intron-length 30 --min-isoform-fraction 0.9 --pre-mrna-fraction 0.25 --overlap-radius 1 --trim-3-avgcov-thresh 0 --trim-3-dropoff-frac 0.0 --small-anchorfraction 0.0

L $\mid$--max-intron-length 4000 --min-intron-length 30 --min-isoform-fraction 0.9 --pre-mrna-fraction 0.50 --overlap-radius 1 --trim-3-avgcov-thresh 0 --trim-3-dropoff-frac 0.0 --small-anchorfraction 0.0

M --max-intron-length 4000 --min-intron-length 30 --min-isoform-fraction 0.9 --pre-mrna-fraction 0.75 --overlap-radius 1 --trim-3-avgcov-thresh 0 --trim-3-dropoff-frac 0.0 --small-anchorfraction 0.0

N --max-intron-length 4000 --min-intron-length 30 --min-isoform-fraction 0.9 --pre-mrna-fraction 0.80 --overlap-radius 1 --trim-3-avgcov-thresh 0 --trim-3-dropoff-frac 0.0 --small-anchorfraction 0.0

0 --max-intron-length 4000 --min-intron-length 30 --min-isoform-fraction 0.9 --pre-mrna-fraction 0.90 --overlap-radius 1 --trim-3-avgcov-thresh 0 --trim-3-dropoff-frac 0.0 --small-anchorfraction 0.0 


\section{Gene predictions with H99 and JEC21 RNA-seq libraries}

201 The validation of this gene prediction system was evaluated by applying the C3Q pipeline with the

best selected Cufflinks parameters (" $Q$ " combination) to all RNA-seq libraries from $C$. neoformans

H99 and C. deneoformans JEC21. For H99, the fifteen libraries obtained from the five growth

conditions were used (Exponential phase at $30^{\circ} \mathrm{C}$, Exponential phase at $37^{\circ} \mathrm{C}$; Stationary phase at

$30^{\circ} \mathrm{C}$, Stationary phase at $37^{\circ} \mathrm{C}$ and Mating). For JEC21, we tested twelve libraries obtained from

four growth conditions (Exponential phase at $30^{\circ} \mathrm{C}$, Exponential phase at $37^{\circ} \mathrm{C}$; Stationary phase at

$30^{\circ} \mathrm{C}$ and Stationary phase at $37^{\circ} \mathrm{C}$.

208 The evaluation of the sensitivity and specificity for gene prediction was performed by comparison 209 of the predicted annotations against H99 and JEC21 reference annotations with the GFFCompare 210 program.

211 For a better understanding, the C3Q pipeline with the " $Q$ " Cufflinks parameters and the RNA-seq

212 libraries for all the sequenced conditions ("ES3037M" for C. neoformans H99 and "ES3037" for C.

213 deneoformans JEC21) is named as C3Q3 protocol in the results section.

$214{ }^{*}$ ES3037M: Exponential phase at $30^{\circ} \mathrm{C}\left(\right.$ EXPO30) + Exponential phase at $37^{\circ} \mathrm{C}(\mathrm{EXPO} 0)+$ Stationary

215 phase at $30^{\circ} \mathrm{C}(\mathrm{STAT30})+$ Stationary phase at $37^{\circ} \mathrm{C}(\mathrm{STAT37})+$ Mating 
*ES3037: Exponential phase at $30^{\circ} \mathrm{C}($ EXPO30 $)+$ Exponential phase at $37^{\circ} \mathrm{C}($ EXPO30 $)+$ Stationary

phase at $30^{\circ} \mathrm{C}(\mathrm{STAT30})+$ Stationary phase at $37^{\circ} \mathrm{C}(\mathrm{STAT37})$

\section{Effect of different conditions on predictions}

219 To evaluate the effect of the growth conditions on the predicted annotation, we used combinations

220 of RNA-seq libraries derived from two, three, and four of the growth conditions for $C$. neoformans

$221 \mathrm{H} 99$ and of two and three of the growth conditions for $C$. deneoformans JEC21. The predictions for

222 each combination were performed according to the $C 3 Q$ pipeline and " $Q$ " Cufflinks parameters. The

223 evaluation of the sensitivity and specificity for gene prediction was performed by comparison of the

224 predicted annotations against $\mathrm{H} 99$ and JEC21 reference annotations with the GFFCompare program.

\section{Evaluation of the effect of the sequencing depth on gene prediction quality}

226 The evaluation of the effect of the sequencing depth on gene prediction was performed by down

227 sampling the three RNA-seq libraries from the EXPO30 condition (replicates A, B, and C) with the 228 tool PositionBasedDownsampleSam from Picard package (https://broadinstitute.github.io/picard/).

229 In this analysis, C. neoformans $\mathrm{H} 99$ and C. deneoformans JEC21 were used. According to a random 230 algorithm that downsamples BAM files, we used defined fractions of 1, 5, 7.5, 10, 15, 20, 30 and 40 231 million reads for each replicate. Subsequently, the predictions were performed according to the 232 C3Q pipeline with the Cufflinks " $Q$ " parameter combination using the downsampled files. Evaluation 233 of the sensitivity and specificity of gene prediction was performed by comparison of the predicted 234 annotations against H99 and JEC21 reference annotations with the GFFCompare program.

\section{Gene predictions with downsampled H99 and JEC21 RNA-seq libraries}

236 Gene prediction using the downsampled BAM files from the RNA-seq conditions was performed 237 according to the $C 3 Q$ pipeline with " $Q$ " Cufflinks parameters and the downsampled RNA-Seq 238 alignment files for all C. neoformans $\mathrm{H} 99$ (Exponential phase at $30^{\circ} \mathrm{C}$, Exponential phase at $37^{\circ} \mathrm{C}$, 
239 Stationary phase at $30^{\circ} \mathrm{C}$, Stationary phase at $37^{\circ} \mathrm{C}$ and Mating) and $\mathrm{C}$. deneoformans JEC21

240 (Exponential phase at $30^{\circ} \mathrm{C}$, Exponential phase at $37^{\circ} \mathrm{C}$; Stationary phase at $30^{\circ} \mathrm{C}$ and Stationary 241 phase at $37^{\circ} \mathrm{C}$ ) growth conditions. The downsampling of each replicate to 7.5 million reads was 242 performed with the Picard package, as previously described. Evaluation of the sensitivity and 243 specificity of gene prediction was performed by comparison of the predicted annotations against $244 \mathrm{H} 99$ and JEC21 reference annotations with the GFFCompare program.

245 For a better understanding, the C3Q pipeline with the " $Q$ " Cufflinks parameters and the subsampled 246 BAM files from RNA-seq libraries for all the growth conditions ("ES3037M" for C. neoformans H99 and "ES3037" for C. deneoformans JEC21) is named as C3Q4 protocol in the results section.

\section{Characterization of novel and missed loci}

249 The identification of novel and missed loci was performed with the GFFCompare program using the 250 reference annotations from C. neoformans H99 and C. deneoformans JEC21 and the predicted C3Q 251 gene annotations. Evaluation of the functional annotation (function, presence of domain signatures) 252 of these sequences was performed by Blastp and Interproscan search from Blast2GO (CONESA et al. 253 2005). The expression quantification of these sequences was performed with HTSeq-count (ANDERS 254 et al. 2014) with the following parameters --stranded yes -f bam -r pos -t CDS.

\section{Deletion of dubious novel loci from predictions}

256 Deletion of dubious novel sequences was tested with predicted transcripts of $100 \mathrm{nt}, 150 \mathrm{nt}, 200 \mathrm{nt}$ 257 and $300 \mathrm{nt}$, as well as intronless sequences of $300 \mathrm{nt}$ from C. neoformans H99 and C. deneoformans 258 JEC21 C3Q predictions. The sequence deletion and evaluation of the results was performed with an 259 in-house AWK script and the GFFCompare program. Deletion of genome-predicted sequences 260 without supporting reads and those with low FPKM values were performed and evaluated with an 261 in-house AWK script combined with the HTSeq-count and the GFFCompare program. Deletion of 262 alternative transcripts from multi-transcript loci was also performed with an in-house AWK script 13 
and GFFCompare. In this process, we selected for transcripts predicted by Cufflinks with supporting

264 RNA-seq evidence. Of these selected transcripts, the longest transcript was chosen. For the other genes predicted only from genome sequencing (without RNA-seq evidence), the longest transcript

We assessed the sensitivity and specificity of the C3Q predictions for $C$. neoformans $\mathrm{H} 99$ and $C$.

268 deneoformans JEC21 against their reference annotations to analyze the effect of dubious sequence

269 deletion. Filter combinations with the low numbers of remnant novel transcripts and smaller

270 reduction in the prediction quality parameters were favored.

271 For a better understanding, the C3Q pipeline with the " $Q$ " Cufflinks parameters, the subsampled BAM from RNA-seq libraries for all the sequenced conditions ("ES3037M" for C. neoformans H99 and "ES3037" for C. deneoformans JEC21), and the sequence filtering (sequences up to $150 \mathrm{nt}$, intronless sequences up to $300 \mathrm{nt}$, genome-predicted sequences without reads and alternative transcripts) is named as C3Q5 protocol in the results section.

\section{Retrieval of deleted and non-predicted loci}

277 The mapping of $C$. neoformans H99 protein sequences in the $C$. deneoformans JEC21 genome and 278 JEC21 protein sequences in the H99 genome by Exonerate v2.2.0 program 279 (https://www.ebi.ac.uk/about/vertebrate-genomics/software/exonerate) with the following parameters ( protein2genome --percent 30 -- bestn 1 --minintron 30 - maxintron 4000 -showalignment false --showvulgar false --showtargetgff true --refine region --subopt false) was

282 performed to recover sequences deleted in the previous filtering step with conserved orthology in 283 Cryptococcus. For this purpose, the mapped gene coordinates matching previously predicted 284 sequences (GFFCompare program) were used to add these deleted genes to the annotation with an 285 in-house AWK script. The addition of non-predicted genes was performed by comparing the mapped 286 protein sequence coordinates and the genomic regions without predicted genes. 
For a better understanding, the C3Q pipeline with the " $Q$ " Cufflinks parameters, the subsampled

BAM from RNA-Seq libraries for all the sequenced conditions ("ES3037M" for C. neoformans H99 and

"ES3037" for C. deneoformans JEC21), the sequence filtering (sequences up to 150 nt, intronless

sequences up to $300 \mathrm{nt}$, genome-predicted sequences without reads and alternative transcripts), and

the Exonerate-based retrieval of deleted and non-predicted genes is named as C3Q6 protocol in the

results section.

\section{Automatization of the C3Q pipeline}

294 The C3Q pipeline, an automatic gene predictor, was built with Python3 code (C3Q_gene-

predictor.py) and is available in Github (https://github.com/UBTEC/C3Q)

The C3Q pipeline includes all established parameters for Cryptococcus genome annotation (C3Q6

297 protocol):

298 - The Cufflinks assembly of transcripts for each RNA-seq library.

299 - The merging of the generated GTF files by Cuffmerge.

300 - The GTF conversion to GFF format (needed for CodingQuarry).

301 - The training and genome prediction by CodingQuarry, using the merged GFF file and the reference 302 genome.

303 - The sequence filtering: deletion of small transcripts up to $150 \mathrm{nt}$ and intronless transcripts up to $304300 \mathrm{nt}$; deletion of genome-predicted sequences without supporting reads and deletion of 305 alternative transcripts from multi-transcript loci.

306 - The retrieval of deleted and non-predicted orthologous/paralogous sequences by Exonerate 307 (modified version with GFF3 support from https://github.com/hotdogee/exonerate-gff3).

308 Gene prediction in C. deuterogattii R265 
309 Gene prediction in C. deuterogattii R265 was performed with the C3Q pipeline (C3Q6 protocol) using

310 the C3Q_gene-predictor.py script. For this, the five RNA-seq triplicate libraries from C. deuterogattii

311 R265 (Exponential phase at $30^{\circ} \mathrm{C}$, Exponential phase at $37^{\circ} \mathrm{C}$, Stationary phase at $30^{\circ} \mathrm{C}$, Stationary

312 phase at $37^{\circ} \mathrm{C}$ and Mating) were subsampled to 7.5 million reads each, and input into the script in

313 addition to the $\mathrm{C}$. neoformans $\mathrm{H} 99$ and $C$. deneoformans JEC21 protein sequences for the Exonerate

314 step.

315 Concomitantly, manual correction of genes from chromosomes 9 and 14 was performed with the

316 software Artemis (CARVER et al., 2012), the R265 genome (NCBI assembly GCA_002954075.1), and

317 the stranded paired-end RNA-seq data from C. deuterogattii R265 in the five growth conditions.

318 The predicted annotation was evaluated by comparing it to the manually corrected genes from

319 chromosomes 9 and 14, as well as the C. deuterogattii R265 annotations from Broad (NCBI assembly

320 GCA_000149475.3) and Ferrareze et al., 2017.

321 CDS gene coordinates from old annotations were also identified in the new sequenced genome with

322 Exonerate aligner (coding2genome). The predicted novel genes were named with CNBG ID numbers 323 above 10000. The statistics of the gene annotations ware generated by AGAT script 324 agat_sp_statistics.pl (https://github.com/NBISweden/AGAT). The final annotation is available in file 325 S1.

326 Comparison of orthologue groups across Cryptococcus species

327 Ortho-groups and genes unique to $C$. neoformans H99, C. deneoformans JEC21 and C. deuterogattii 328 R265 were evaluated with Orthofinder v2.3.3 configured to use the Blast aligner. Gene size 329 comparisons were performed with orthologues and paralogues (if the true orthologue was not 330 known) obtained from the OrthoFinder analysis (EMMS AND KELLY 2019), as well as gene sizes. For the 331 ratio calculation, the size (nt) of the R265 gene was divided by the size (nt) of the H99 and JEC21 332 orthologous genes. The analysis of conserved domains in unique sequences and the functional 16 
333 annotation of $C$. deuterogattii R265 were performed with Blast2GO (Blastp, Interproscan and GO

334 mapping).

\section{Gene orientation analysis}

336 To determine the frequency of tandem genes with the same orientation, we searched for groups of

337 two, three, four, or five genes assigned to the same DNA strand in the GFF file from C. deuterogatti 338 R265 (with our new annotation), C. neoformans H99 (Genome assembly reference

339 GCF_000149245.1), and C. deneoformans JEC21 (Genome assembly reference GCF_000091045.1)

340 annotations. This was performed by analyzing the orientation of each gene pair in the GFF file from

341 R265, H99 and JEC21, recording the frequency of genes converging (tail-to-tail), diverging (head-to-

342 head), and in the same orientation (head-to-tail) in the whole genome and for each chromosome.

343 This was executed using an in-house Python script (script gene_organization_analysis.py).

\section{Antisense transcription analysis}

345 To evaluate the antisense transcription in the genomes analyzed, we first generated a reversed 346 annotation, which consisted of a GFF file with genes assigned to the opposite strand of their actual 347 annotation. With the annotation and the reverse annotation, we analyzed the percentage of 348 antisense transcription for each protein coding gene using the software HTseq-count using the 349 following attributes (- $f$ bam $-r$ pos - $s$ yes $-t$ CDS -i ID -m intersection-nonempty --nonunique none 350 (.bam) (.gff)) and the distinct RNA-seq libraries. Sense/antisense counts ratios for each gene for each 351 condition were plotted. The script used for generation of a reverse GFF is available (reverse_gff.py).

\section{Intron retention evaluation}

353 For a given intron, an IR index was calculated by determining the ratio of spliced:non-spliced reads 354 at the upstream and downstream exon-intron junctions and choosing the lowest of these two 355 numbers. These IR indices were calculated using RNA-seq obtained from cells growing in each of the 
356 four growth conditions. An intron was considered to be regulated by intron retention when the IR

357 index was at least 0.01 . We restricted our analysis to introns with more than 10 spliced reads.

\section{Statistical analysis}

359 The proportion of genes with intron retention regulation was compared amongst the distinct 360 conditions using one way ANOVA followed by multi-comparison analysis corrected by FDR. The X361 squared analysis was conducted using R (version 4.0.2) and plots were prepared using the corrplot 362 package (version 0.84).

\section{Availability and accession number}

364 Raw and summarized sequencing data are available at SRA with the accession number: 365 PRJNA660459. The C3Q pipeline is available in Github (https://github.com/UBTEC/C3Q); 366 Supplemental files available at FigShare. The final annotation of $\mathcal{C}$. deuterogattii genome was 367 submitted to NCBI and is available on accessions CP025759.1 to CP025772.1.

RESULTS

\section{Pipeline selection}

We first compared the performance of two previously published annotation pipelines used

372 in coding gene de novo annotation in intron-rich fungal genomes using RNA-seq data. The

373 BRAKER1 pipeline, which combines GeneMark-ET (LOMSADZE et al. 2014) and Augustus (STANKE et

374 al. 2008) and is already optimized with the best prediction parameters (Hoff et al. 2016), was

375 compared with a genome annotation pipeline composed of Cufflinks v2.1.1 (TRAPNELL et al. 2010)

376 and CodingQuarry v2.0 (TESTA et al. 2015). We used the C. neoformans H99 and C. deneoformans

377 JEC21 genomes as controls to assess of the performance of both pipelines (GonZALEZ-HILARION et al. 378 2016; WALLACE et al. 2020). 
For this analysis, we used only RNA-seq data obtained in biological triplicate from cells

grown to exponential growth phase at $30^{\circ} \mathrm{C}$ in complete medium (YPD) (EXPO30) (WALLACE et al.

381 2020). Previously described BAM files obtained after alignment of trimmed reads to the $C$.

382 neoformans H99 genome were input into the BRAKER1 and Cufflink-CodingQuarry pipelines

383 (WALLACE et al. 2020). For the Cufflink-CodingQuarry-based analyses, we used two alternative

384 protocols. In the first case, we first merged the BAM files from each of the three replicates (CUFF-

385 CQ protocol). In the second case, each replicate BAM file was used to generate a unique GTF

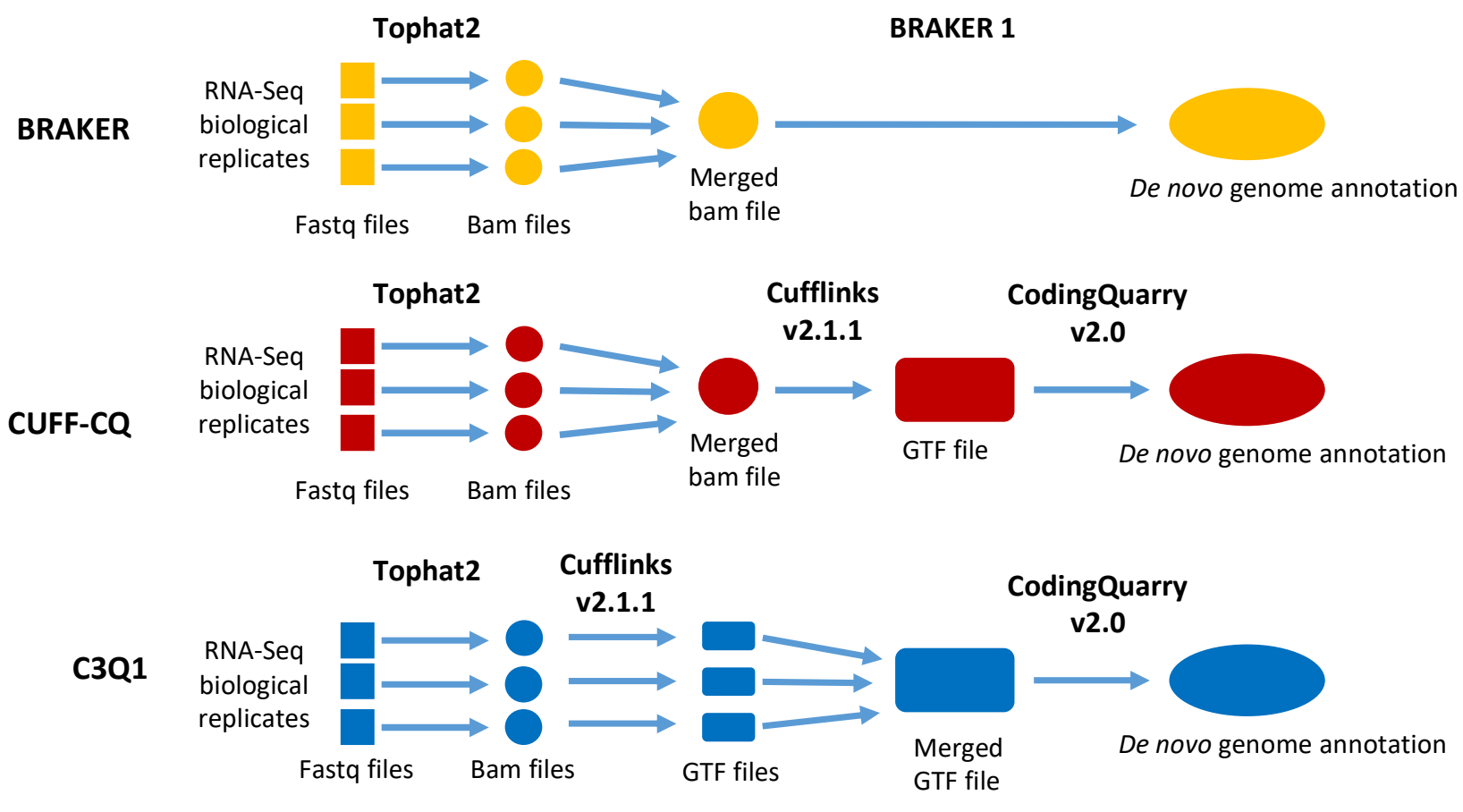

390 Figure 1. Schematic of the different pipelines tested in this study. 
genes overlapping with one coding gene in the reference annotation). These comparisons revealed that BRAKER was much more sensitive than either Cufflinks-CodingQuarry protocol, missing only

91 coding genes in the $C$. neoformans genome (Figure 2A, Supplementary Table S1). However, the BRAKER pipeline was less specific (91.4\%), predicting 622 coding genes absent in the reference annotation (Figure 2B). In contrast, both Cufflink-CodingQuarry protocols missed more coding genes ( 743 and 447 genes for CUFF-CQ and C3Q protocols, respectively), but had a higher (95\%) specificity (Figures 2A,2B). We observed a similar pattern when we looked at CDS introns and CDS exons within the identified references genes. Again, the BRAKER pipeline was very sensitive, with only $0.4 \%$ missed introns $(n=157)$ and $0.4 \%$ missed exons $(n=164)$ in the prediction but had poor specificity, with 4471 novel introns and 3065 exons predicted but not present in the reference

protocols missed more introns ( $n=4944$ and $n=3238$ for CUFF-CQ and C3Q1 protocols,

respectively) and more exons ( $n=4281$ and $n=2777$, respectively) but both were more specific, specific in the predicted gene structures and identified a smaller number of new insertions (novel exons/introns) and new genes. These more conservative predictions came at the cost of missing a larger number of features than the BRAKER protocol.

414 for further optimization, we considered the sensitivity and specificity of accurately predicting gene 415 structure (perfect exon/intron organization) for each of the three pipelines. The C3Q1 protocol 416 was the most sensitive, perfectly predicting the exon-intron layout of $66.5 \%(n=4516)$ of $C$.

417 neoformans $\mathrm{H} 99$ genes, compared to $65.2 \%$ and $51.9 \%$ perfect predictions from the BRAKER and 418 CUFF-CQ protocols, respectively (Figure 2C). This was also the most specific protocol with $63.9 \%$ of 419 the predicted genes perfectly fitting the reference gene structures, compared to $61.3 \%$ and $52.6 \%$ 
of the predictions made by the BRAKER and CUFF-CQ protocols, respectively (Figure 2C). To better compare the quality of these pipelines, we considered a quality index that multiplied the sensitivity by the specificity of predicted gene structure predictions (Figure 2D). Our results demonstrated that the C3Q1 pipeline was the best, with a quality index of 0.42 . We performed the same analysis with the $C$. deneoformans JEC21 genome annotation data and obtained similar results, confirming the C3Q1 protocol was the best protocol for further optimization (Figure S1).
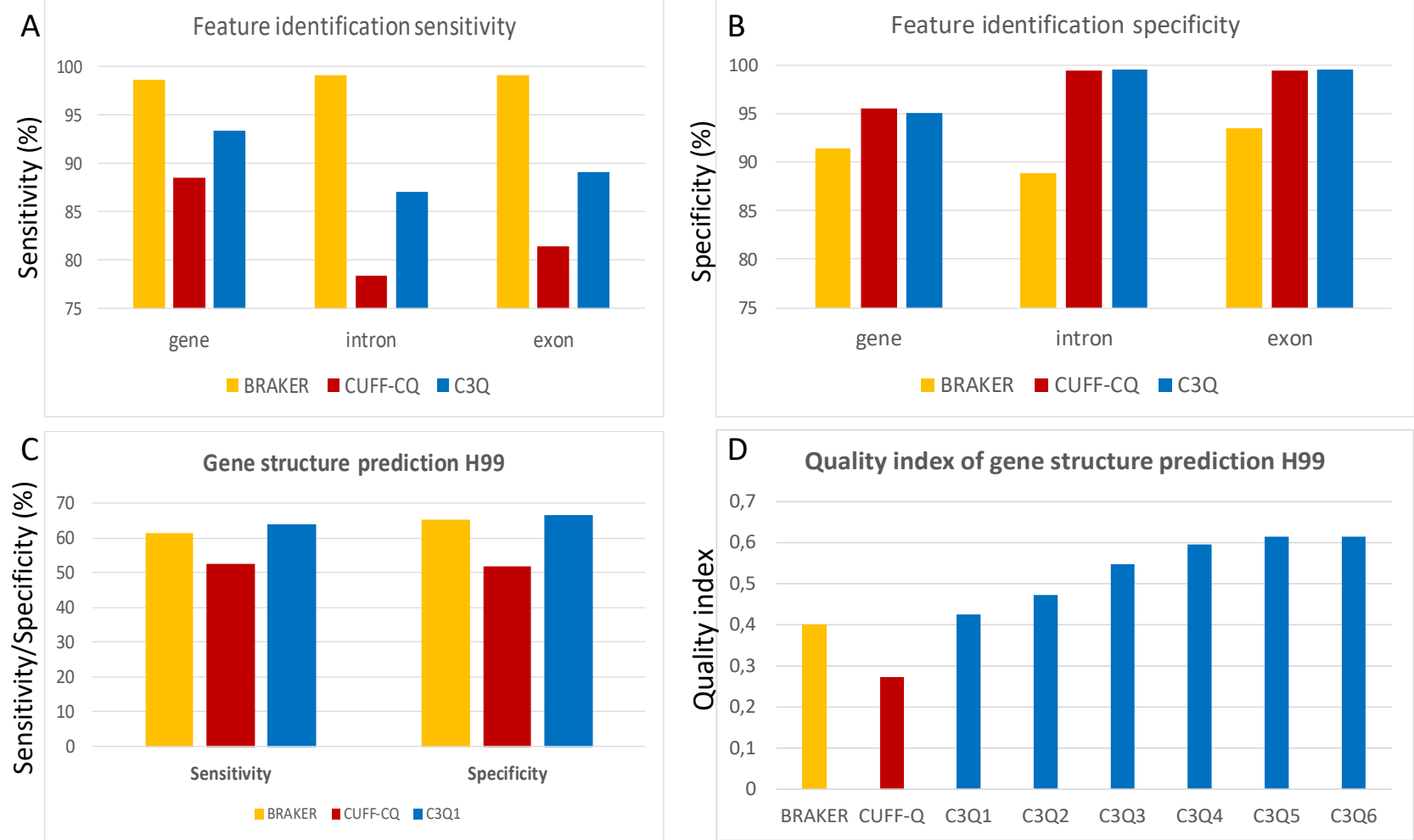

Figure 2: Sensitivity (A) and specificity (B) of the different tested pipelines for C. neoformans H99 genomic feature identification. For introns and exons, calculations were done using only genes that were both identified by the pipelines and present in the reference annotation. (C) Sensitivity

431 and specificity of gene structure predictions using the three annotation pipelines. (D) Optimization 432 of the $C 3 Q$ pipeline. $C 3 Q 1$ is the pipeline using default settings. C3Q2 through C3Q6 refer to the 433 results obtained after each step of the pipeline optimization. 


\section{Optimization of the C3Q1 pipeline}

\section{Effect of Cufflinks settings}

To improve both the number of perfectly predicted gene structures and the percentage of predicted loci in perfect agreement with the reference coding gene structures, we considered 17 combinations of Cufflinks settings. We varied parameters including 1) the minimum distance between transfags allowed to merge, 2) trimming of 3' ends of reads, 3) filtering of alignments that lie within intronic intervals, 4) filtering of suspicious spliced reads, 5) minimum RNA-seq fragments allowed to assemble transfags, and 6) filtering of alignments that lie within intronic intervals in the same set of RNA-seq data. These Cufflinks parameter modifications reduced the number of missed genes and increased the number of reference genes identified from 6348 to 6462 (Table S1). Using the final settings, the pipeline C3Q2 quality index reached a score of 0.473 , with $70.8 \%$ of reference gene intron-exon structures perfectly predicted and $66.8 \%$ of the predicted genes perfectly matching the reference exon-intron gene structures (Figure 2D).

\section{Effect of the RNA-seq data set}

We tested the C3Q2 optimized pipeline using additional RNA-seq data obtained under five different conditions in triplicate: stationary growth at $30^{\circ} \mathrm{C}$ (STAT30) and $37^{\circ} \mathrm{C}$ (STAT37), exponential growth at $30^{\circ} \mathrm{C}(\mathrm{EXPO} 30)$ and $37^{\circ} \mathrm{C}$ (EXPO37), and growth under mating conditions (Mating). Each RNA-seq data set generated a similar number of predicted transcripts, ranging between 7049 genes using the STAT37 set up to 7199 loci using the EXPO30 data set (Table S1). When compared to the reference set of genes, the number of predicted annotations were also very similar (Table S1). As expected, including more samples improved the annotation quality. The usage of the five conditions improved the C3Q3 annotation quality index to 0.547 despite the fact that more predicted genes not present in the reference genome were identified using this pipeline 
bioRxiv preprint doi: https://doi.org/10.1101/2020 09.01 278374 this version posted December 14,2020 . The copyright holder for this preprint (which was not certified by peer review) is the author/funder, who has granted bioRxiv a license to display the preprint in perpetuity. It is made available under aCC-BY-NC-ND 4.0 International license.

459 ( $n=510)$ (Figures 2D, S2, Table S1). Similar results were obtained using the $C$. deneoformans JEC21 460 annotation and RNA-seq data (Figure S1, Table S1). 
missed.
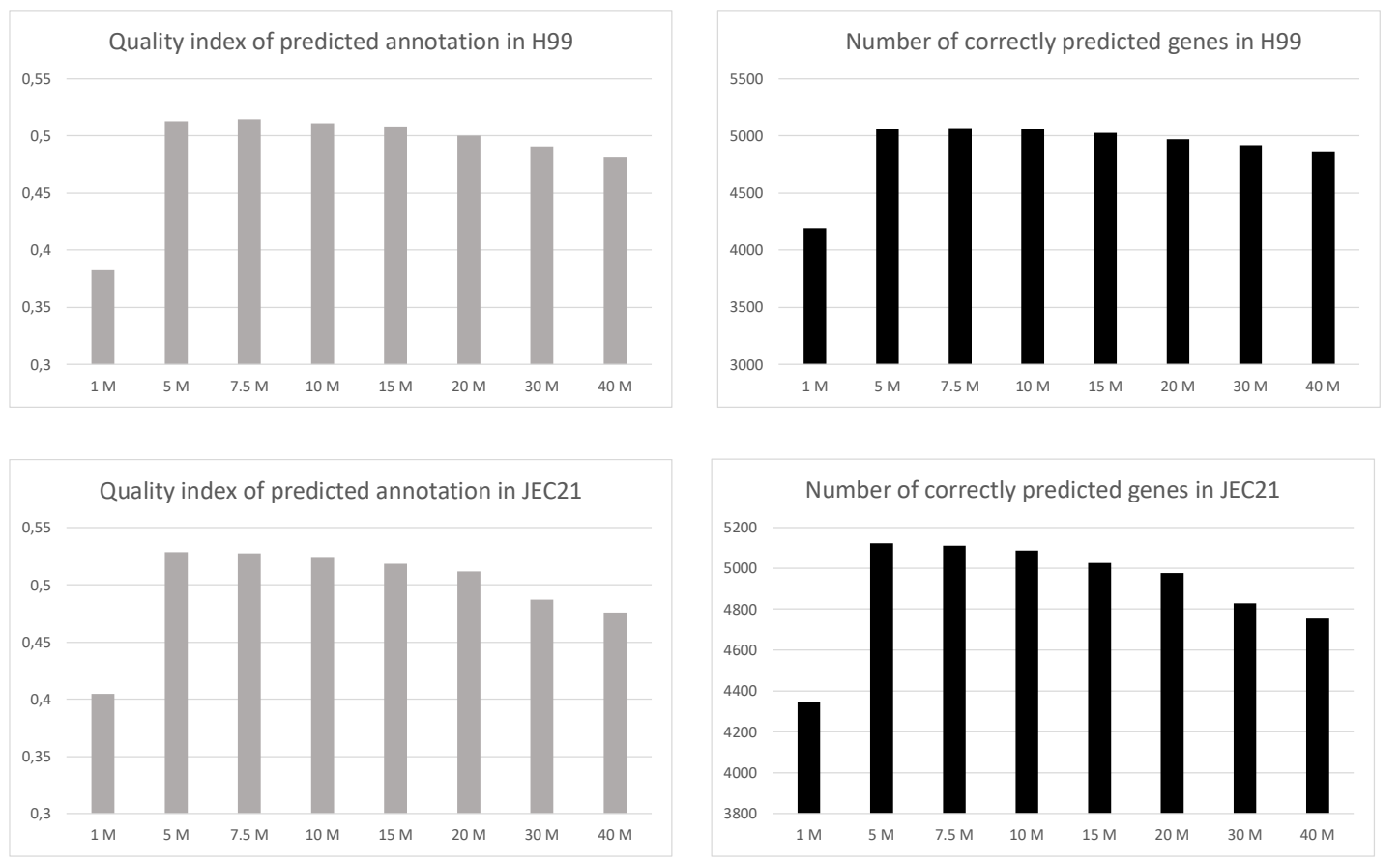

Figure 3. Effect of the size of the BAM file on the quality of the predicted annotation and on

the number of correctly predicted genes in C. neoformans H99 and C. deneoformans JEC21.

Gene filtering

Each optimization step improving the quality of the gene prediction was also associated

with an increase of the number of predicted genes not present in the previously annotated and six of the sequences had domain signatures of transposable elements, suggesting they 
coverage. We compared the characteristics of these false-positive genes to the reference genes and found that most novel predicted genes were short (219 nt mean length, $112 \mathrm{nt}$ median

501 length), poorly expressed, and intronless. We tested different filters alone and in combination to eliminate as many false positive genes as possible without affecting the number of correctly predicted ones; the results are presented in Table S1. In both species, the best combination of

504 filters eliminated all spliced coding regions smaller than $150 \mathrm{nt}$, all intronless genes smaller than $300 \mathrm{nt}$, and all genome-predicted genes not supported by any RNA-seq reads. Due to the presence of secondary transcripts at some loci, many of which were generated due to differences in the

evidence) was selected as a representative for the gene CDS coordinates. After this fourth filtering step, the number of predicted genes not present in the reference annotation was down to 409 and

427 genes in H99 and JEC21, respectively, and the quality index of the annotation increased to H99 genes and 132 JEC21 genes were missed with the C3Q5 pipeline. Blast2GO analysis of the protein sequences encoded by the missed genes identified 16 proteins with conserved domains

519 suggesting that it might be possible to identify some of them through comparative sequence analysis. Another 111 sequences were defined as hypothetical proteins. We first used the sequence alignment program Exonerate (SLATER AND BIRNEY 2005) and the JEC21 reference 
523 identified a number of missed loci, but also added a number of unpredicted loci thus reducing the

524 quality of the annotation. In the final C3Q6 pipeline, we chose to restrict this Exonerate analysis to 525 genes that had been filtered out in the last step of the C3Q5 pipeline. We ultimately identified 14

526 and 9 novel genes in $\mathrm{H} 99$ and JEC21, respectively. Overall, the C3Q6 optimized pipeline was able to 527 identify nearly $98 \%$ of genes in $\mathrm{H99}$, contributing only 410 ( 6\%) novel genes. Importantly, the 528 exon-intron structure of the predicted genes was predicted perfectly for $>81 \%$ of the reference 529 genes in both species.

\section{Intron number is predictive of the quality of the C3Q6 predicted annotation}

534 looking for a parameter predictive of their quality. First, we plotted the number of predicted loci against the quality of the annotation, but we did not observe any correlation. Similar results were obtained when we looked at missed or novel loci, suggesting that these parameters were also not indicative of the annotation quality. However, when the numbers of introns predicted were plotted against the quality of the annotation, we obtained a linear correlation (Figure 4). This correlation was lost during the filtration steps (red dots), which tend to reduce the number of parameter to consider when evaluating the quality of the annotation using the C3Q pipeline. 


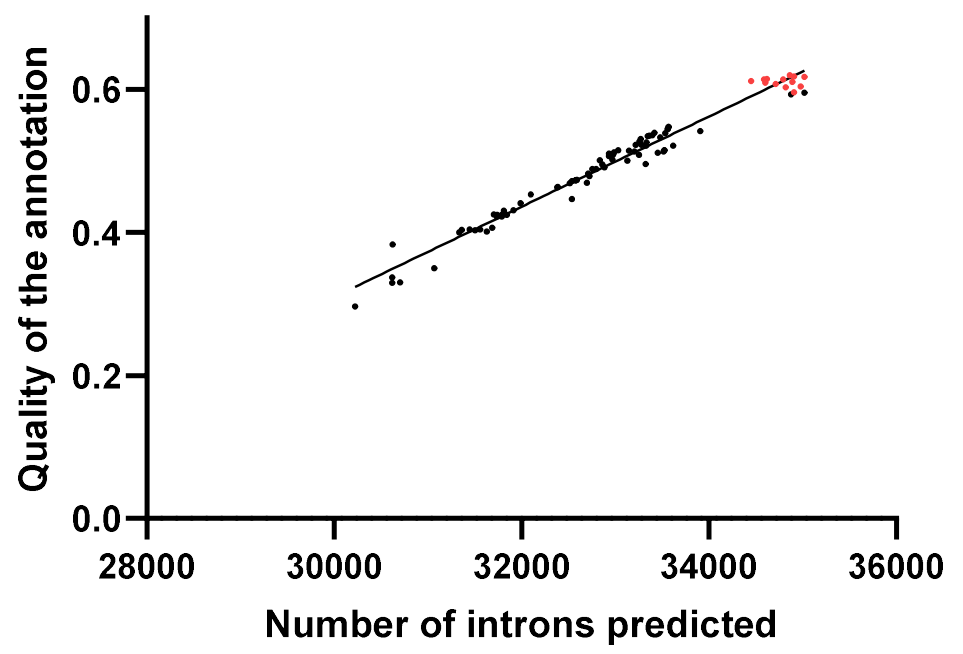

543 Figure 4. Relationship between the quality index of the $\mathrm{H} 99$ annotation and the number of introns 544 and transcripts predicted. The red points correspond to the filtering steps of the optimization 545 pipeline.

\section{Genome annotation of the Cryptococcus deuterogattii genome}

We used the C3Q6 optimized pipeline to generate a new genome annotation for the $C$.

551 deuterogattii reference strain R265. This reference strain was isolated in 2001 from the

552 bronchoalveolar lavage fluid of an infected patient from the Vancouver Island outbreak (KIDD et al.

553 2004). Because of its outbreak origin and the loss of a functional RNAi pathway (D'SouzA et al.

554 2011), C. deuterogattii has been the focus of a number of studies in recent years (CHENG et al.

555 2009; Ma et al. 2009; NgAMSkULRUngros et al. 2012; Huston et al. 2013; LAM et al. 2019). The R265

556 genome has been previously annotated three times (D'SOUZA et al. 2011; FARRER et al. 2015;

557 FERRAREZE et al. 2017), but a recent release of telomere-to-telomere genome sequence data (YADAV 558 et al. 2018) motivated us to generate an updated annotation. We generated RNA-seq data in 559 biological triplicate from cells grown under five conditions (exponential growth phase at $30^{\circ} \mathrm{C}$ and 
$37^{\circ} \mathrm{C}$, stationary growth phase at $30^{\circ} \mathrm{C}$ and $37^{\circ} \mathrm{C}$, and under mating conditions) as previously

described for C. neoformans H99 and JEC21 (WALLACE et al. 2020). Reads were trimmed, aligned to

the reference genomes (Table S2), and input into the optimized C3Q6 genome annotation

563 pipeline.

To gain further insight into the quality of our updated R265 annotation, the structure

predictions of genes for $C$. deuterogattii R265 chromosomes 9 and 14 were manually curated

through visual examination of read alignments using Artemis (CARVER et al. 2012) as previously

described (JANBON et al. 2014; GonZALEZ-HILARION et al. 2016). We compared this manually curated

curated genes on these two chromosomes correctly predicted. In C. neoformans and $C$.

C3Q6 annotation was much better than previously published annotations (FARRER et al. 2015;

FERRAREZE et al. 2017) (Table S3).

\section{Manual curation of R265 annotation}

To systematically analyze critical points of the automated C. deuterogattii R265 gene

prediction, four sets of data were evaluated and selected for manual correction: 1) Exonerate-

retrieved sequences (deleted and non-predicted), 2) predicted novel loci, 3) genes predicted in

merged/split loci, and 4) small and potential pseudogenes. During the course of this manual 
or splitting and might partially explain the lower quality index score calculated for the R265

predicted annotation of chromosomes 14 and 9 compared to the quality scores obtained using

similar data from JEC21 and H99. To systematically identify these types of annotation mistakes, we

compared the size of the $C$. deuterogattii R265 predicted genes with their $C$. neoformans $\mathrm{H} 99 / \mathrm{C}$.

590 Visual examination of these loci revealed that most of them were incorrectly predicted and

591 needed manual curation. Manual curation was also performed for 67 genes that were significantly curation also identified 125 genes which would have otherwise been challenging to predict due to genome sequence errors mistakenly affecting orthologue size ratios (Table S4). and/or the transcript leader sequence was performed for 1210 genes from the manually curated

601 chromosomes ( 9 and 14) and the 873 manually curated genes with modified CDS structures.

602 Furthermore, we annotated 55 IncRNAs and used tRNAscan (LOWE AND CHAN 2016) to annotate 161

603 tRNAs (Table S3). We also removed all genes predicted to reside within centromeric regions, and 604 used previously published, manually curated annotations for these regions (YADAV et al. 2018).

\section{Putative pseudogenes and missing genes}



an indicator of the high quality of this annotation. Of interest, this analysis revealed 210 ortho-

611 groups missing in $C$. deuterogattii R265, but present in both the $C$. neoformans $\mathrm{H} 99$ and the $C$.

612 deneoformans JEC21 genomes (Table S5). This list of genes was curated first through Exonerate-

613 based analysis and then through manual examination of the syntenic loci.

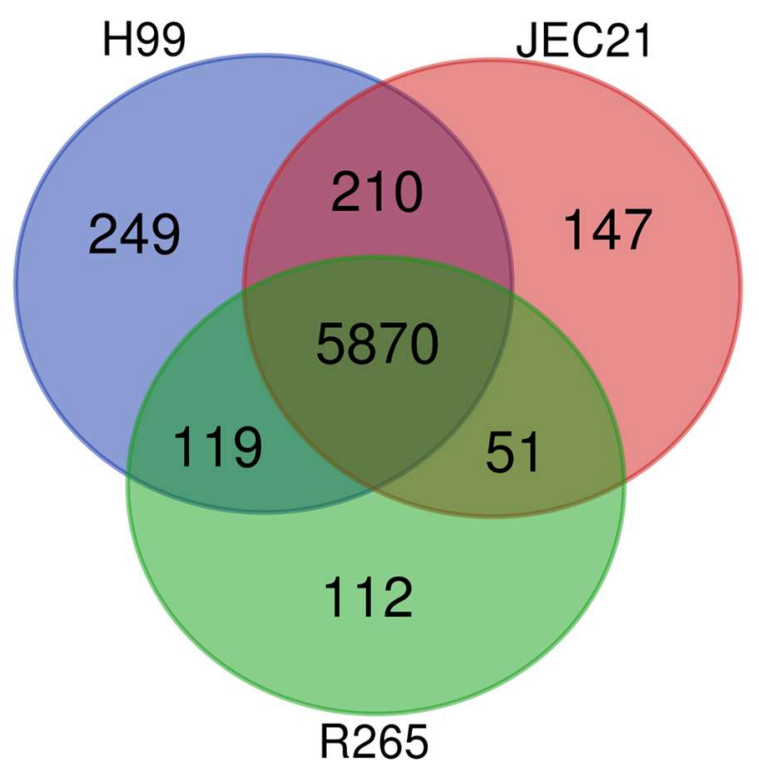

616 Figure 5. Comparative gene content of the annotated C. neoformans H99, C. deneoformans JEC21, and $C$. deuterogattii R265 genomes. Ortho-groups specific or common to the different species were identified and numbered. pathway (D'SOUZA et al. 2011; BILLMYRE et al. 2013). Accordingly, the genes encoding one Dicer (DCR1) and an Argonaute protein (AGO1) have been lost, and the genes encoding an RNA 
identification of truncated or absent genes in the R265 genome has been as a strategy to identify additional, novel components of the RNAi pathway in C. neoformans (FERETZAKI et al. 2016). To identify genes specifically lost in C. deuterogattii, we considered the genes not predicted by our pipeline but present in the other Cryptococcus species annotations available in FungiDB (BASENKO 
bioRxiv preprint doi: https://doi.org/10.1101/2020.09.01.278374; this version posted December 14, 2020. The copyright holder for this preprint (which was not certified by peer review) is the author/funder, who has granted bioRxiv a license to display the preprint in perpetuity. It is made available under aCC-BY-NC-ND 4.0 International license.

\begin{tabular}{|c|c|c|c|c|c|}
\hline H99 gene ID & $\begin{array}{l}\text { R265 gene } \\
\text { ID }\end{array}$ & $\begin{array}{l}\text { Size } \\
\text { ratio }\end{array}$ & Putative function & $\begin{array}{l}\text { Role in } \\
\text { RNAi }\end{array}$ & ref \\
\hline CNAG_00505 & absent & & transcription factor (FZC28) & yes & $(1)$ \\
\hline CNAG_01061 & absent & & serine/threonine protein kinase (FRK102) & $?$ & $(2)$ \\
\hline CNAG_02207 & absent & & glycosyl hydrolase & $?$ & \\
\hline CNAG_03734 & absent & & chromodomain-containing protein (CDP1) & $?$ & $(1)$ \\
\hline CNAG_04016 & absent & & Identified spore protein 5 (ISP5) & $?$ & (3) \\
\hline $\begin{array}{l}\text { CNAG_04596 } \\
\text { CNAG_04619 }\end{array}$ & absent & & prolyl endopeptidase & $?$ & \\
\hline CNAG_04609 & absent & & Argonaute protein $(A G O 1)$ & yes & $(4)$ \\
\hline CNAG_05158 & absent & & hypothetical protein & $?$ & - \\
\hline CNAG_05265 & absent & & hypothetical protein & $?$ & \\
\hline CNAG_05449 & absent & & copper metallothionein 1 (MTN1) & $?$ & (5) \\
\hline CNAG_05657 & absent & & 2,4-dienoyl-CoA reductase & $?$ & \\
\hline CNAG_06233 & absent & & hypothetical protein & $?$ & \\
\hline CNAG_06395 & absent & & hypothetical protein & $?$ & \\
\hline CNAG_06609 & absent & & $\begin{array}{l}\text { 2-polyprenyl-6-methoxyphenol hydroxylase } \\
\text { (ORX1) }\end{array}$ & $?$ & (1) \\
\hline CNAG_07556 & absent & & hypothetical protein & $?$ & \\
\hline CNAG_07702 & absent & & F-box containing protein & $?$ & \\
\hline CNAG_07959 & absent & & GTPase-activator protein (GAP) & $?$ & \\
\hline CNAG_03466 & CNBG_2143 & 0.083 & RNA-dependent RNA polymerase 1 (RDP1) & yes & $(4)$ \\
\hline CNAG_02700 & CNBG_9326 & 0.092 & $\begin{array}{l}\text { C2H2-type zinc finger transcription factor } \\
(Z N F 3)\end{array}$ & yes & (1) \\
\hline CNAG_01423 & CNBG_5946 & 0.102 & QIP1 & yes & $(6)$ \\
\hline CNAG_04146 & CNBG_2894 & 0.121 & SET domain-containing protein & $?$ & \\
\hline CNAG_06486 & CNBG_4982 & 0.137 & GWC1 & yes & $(6)$ \\
\hline CNAG_03911 & CNBG_9603 & 0.139 & carboxylesterase domain-containing protein & $?$ & \\
\hline CNAG_06497 & CNBG_4974 & 0.162 & microsomal epoxide hydrolase (MEH1) & $?$ & $(1)$ \\
\hline CNAG_01992 & CNBG_2960 & 0.190 & SET domain-containing protein & $?$ & \\
\hline CNAG_03117 & CNBG_2464 & 0.191 & hypothetical protein & $?$ & \\
\hline CNAG_07344 & CNBG_9031 & 0.197 & Ras guanyl-nucleotide exchange factor & $?$ & \\
\hline CNAG_01406 & CNBG_5961 & 0.198 & hypothetical protein & $?$ & \\
\hline CNAG_03414 & $\begin{array}{l}\text { CNBG_1006 } \\
4\end{array}$ & 0.201 & $\begin{array}{l}\text { REX4-like exonuclease domain containing } \\
\text { protein }\end{array}$ & $?$ & \\
\hline CNAG_04184 & CNBG_2860 & 0.224 & transcription factor (FZC47) & no & $(1)$ \\
\hline CNAG_03193 & CNBG_9232 & 0.233 & hypothetical protein & $?$ & \\
\hline CNAG_04400 & CNBG_9268 & 0.234 & Ribosomal protein S10p/S20e & $?$ & \\
\hline CNAG_03938 & CNBG_5530 & 0.243 & Cryptococcus pheromone receptor 2 (CPR2) & yes & $(1)$ \\
\hline CNAG_00123 & CNBG_9042 & 0.286 & hypothetical protein & $?$ & \\
\hline CNAG_06159 & CNBG_4866 & 0.315 & hypothetical protein & $?$ & \\
\hline CNAG_01004 & CNBG_0584 & 0.316 & Rho/Rac/Cdc42-like GTPases & $?$ & \\
\hline CNAG_06509 & CNBG_4963 & 0.318 & hypothetical protein & $?$ & \\
\hline
\end{tabular}

Table 2: Genes with putative or known roles in RNAi identified as genes of H99 with orthologues in all Cryptococcus species but absent or severely truncated (and thus putative pseudogenes) in C. deuterogattii R265 as compared to JEC21 and H99 (proteins with a ratio $<0.33$ are presented. The full table of shortened genes is presented as Table S6). (1) FERETZAKI et al 2016; (2) Lee et al. 2016; (3) HuANG et al. 2015; (4) WANG et al. 2010 ; (5) Ding et al. $2011 ;(6)$ DuMESIC et al. 2013. 
660 (BURKE et al. 2019) were present in this list, confirming that a large number RNAi genes are not

661 functional or are absent in R265. Conversely, RDE1, RDE2, and RDE3 (BURKE et al. 2019), which

662 were recently implicated in RNAi in C. neoformans, all possess an orthologue of similar size in R265

663 (CNBG_3369, CNBG_4718, and CNBG_1922, respectively). Of note, in this version of the R265

664 annotation, the DMT5 (CNBG_3156) gene encoding a putative DNA methyltransferase is not

665 truncated as previously published (YADAV et al. 2018; CATANIA et al. 2020) and appears to be 666 expressed and functional.

\section{Gene organization, antisense transcription, and alternative splicing in R265}

the chromosome structure: shorter centromeres and the loss of any full-length transposable elements (YADAV et al. 2018). Here, we examined the possible consequences of RNAi loss on the source of double-stranded RNA; increased antisense transcription in RNAi-deficient species has also been observed in Saccharomyces species (ALCID and TSUKIYAMA 2016). We thus evaluated the sense/antisense transcript ratio at coding gene loci. Because the 3'UTR and TL sequences were only partially annotated in the R265 genome, we restricted our analysis to the CDS regions. We compared the ratio of read numbers of sense and antisense transcripts corresponding to all coding 
681 (92.2\%) had antisense transcription in C. neoformans, but antisense transcripts were expressed at

682 a very low level (1.2\% of transcription antisense vs sense). Antisense transcription prevalence and 683 expression levels were similar in the two other species (92.6\% and $95.2 \%$ of genes with antisense transcription, $3.2 \%$ and $2.5 \%$ of antisense vs sense transcription in $C$. deneoformans and $C$. increased temperature at both exponential and stationary growth phase. However, this analysis did not provide evidence of a link between the level of antisense transcription and the absence of RNAi in R265 because the RNAi-proficient JEC21 strain had the highest antisense/sense transcription ratio across all conditions tested (Figure 6).

We then analyzed gene orientation in the three species, evaluating the number of genes coupled in a tail-to-tail orientation as this orientation should favor antisense transcription over head-to-tail or head-to-head orientations. Indeed, as shown in Figure 6B, there was a clear selection against tail-to-tail gene orientation in C. deuterogattii, thus limiting antisense transcription (c-squared $=103.79, \mathrm{df}=4, \mathrm{p}$-value $<2.2 \mathrm{e}-16$ ). In contrast, this orientation is favored in JEC21, which might explain the higher level of antisense transcription. 
value of the IR index in R265 was higher than those in both $C$. neoformans and $C$. deneoformans.

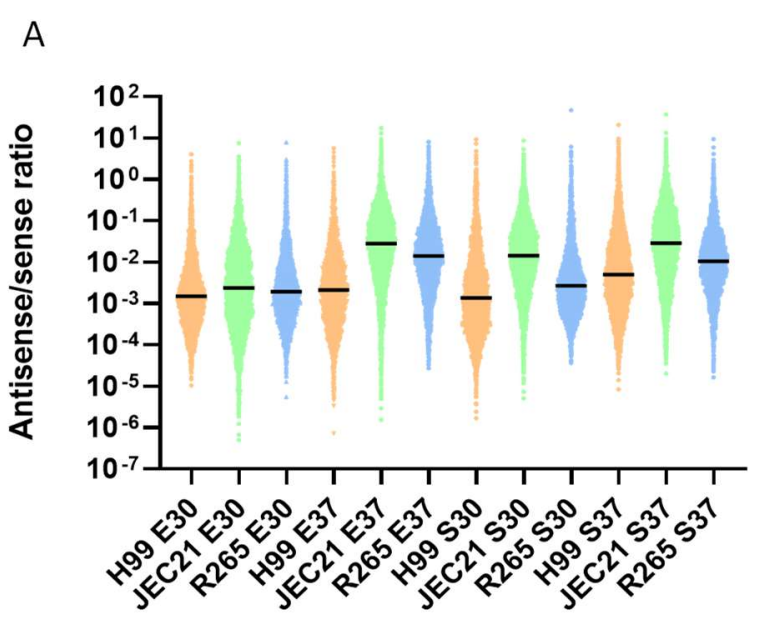

Strains and growth conditions

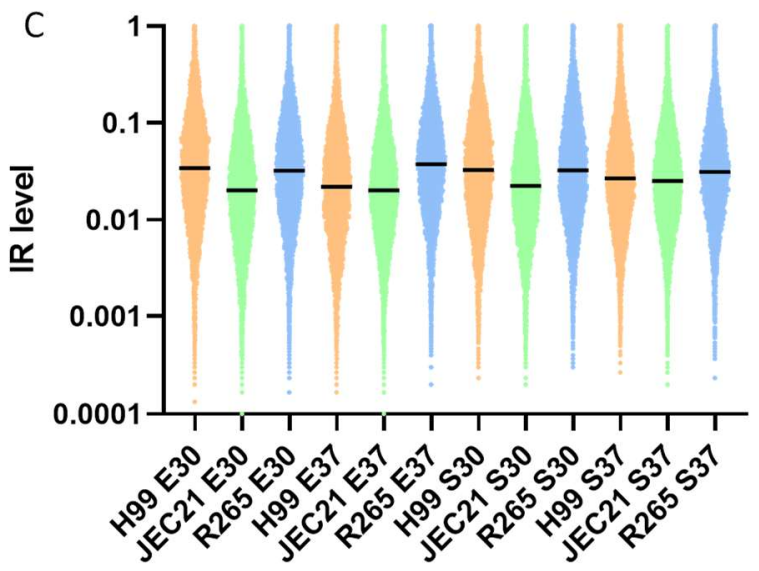

Strains and growth conditions

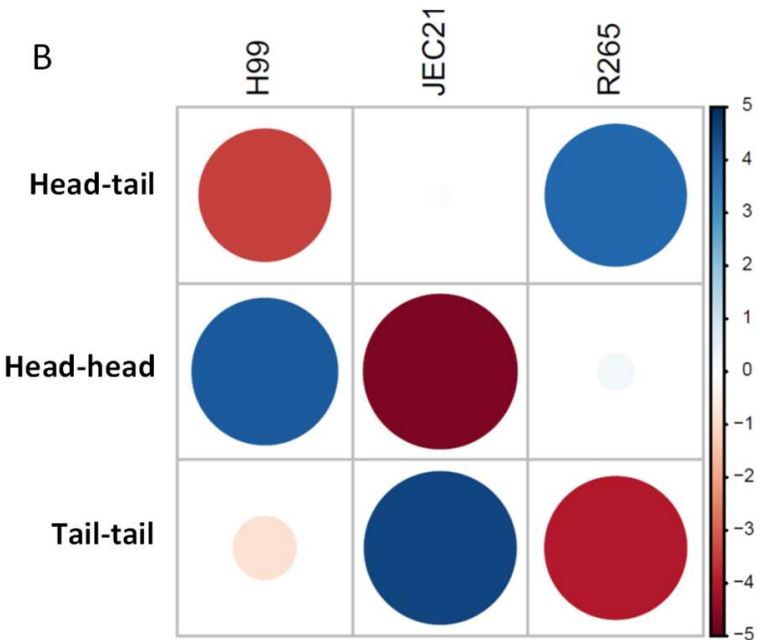

D

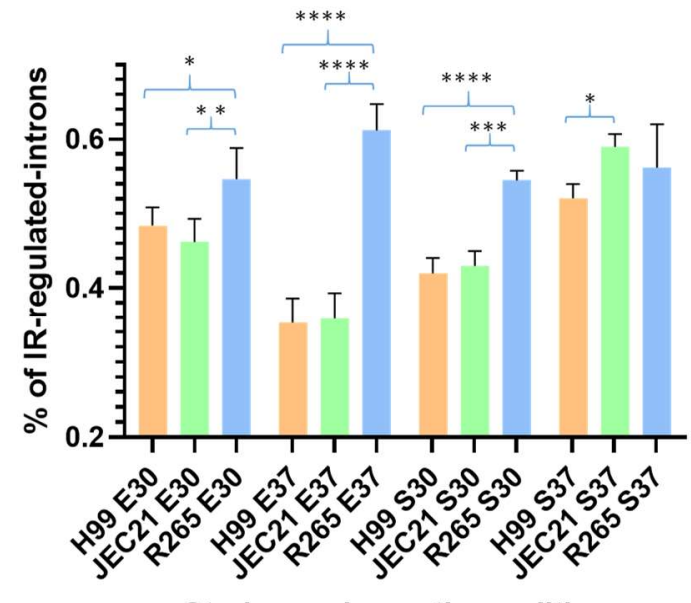

Strains and growth conditions

711 Figure 6. (A) Antisense/sense transcription ratios in C. neoformans (H99), C. deneoformans (JEC21)

712 and C. deuterogattii (R265). RNA-seq data obtained from cells grown to exponential phase at $30^{\circ} \mathrm{C}$

$713(\mathrm{E} 30)$ and $37^{\circ} \mathrm{C}(\mathrm{E} 37)$ or stationary phase at $30^{\circ} \mathrm{C}(\mathrm{S} 30)$ and $37^{\circ} \mathrm{C}(\mathrm{S} 37)$ were used. (B) Statistical

714 analysis (Pearson's Chi-squared test) revealed a species-specific bias in gene orientation. Circle size

715 is proportional to the standardized residuals, with absolute values higher than 2 representing

716 statistical significance (Sharpe 2015). Positive values (blue circles) in cells specify a positive 
717 association between the corresponding row and column variables. Negative residuals are

718 represented by red circles. This implies a negative association between the corresponding row

719 and column variables. (C) Intron retention level in each species according to growth condition.

720 Black bars represent median values. (D) Percentage of CDS introns regulated by IR in each species

721 according to growth condition. The results of the statistical analysis (ANOVA one-way multi-

722 comparison analysis corrected by FDR). * (q value $<0.05), * *$ (q value $<0.01$ ), *** q value $<0.001$ ),

$723 * * * *$ (q value $<0.0001)$

\section{Subtelomeric gene organization and cluster exchange in Cryptococcus}

Our analysis identified 210 orthologue groups present in both $C$. neoformans and $C$.

deneoformans but absent in C. deuterogattii. Interestingly some of these lost genes are clustered

in these genomes. We identified 21 clusters of lost genes with consecutive elements in both $C$.

neoformans and $C$. deneoformans reference genomes. One of these lost clusters has been

previously described and has been reported to contain homologues of several GAL genes (GAL1,

731 UGE2, and GAL7) and a gene encoding a sugar transporter of the major facilitator superfamily

732 (MFS) (CNAG_07897) (SLOT AND ROKAS 2010). We also identified a fifth gene in this cluster

733 (CNAG_06055) which encodes a putative alpha-1,4-galactosidase (Figure S2A). C. neoformans and

734 C. deneoformans also possess unclusterered paralogues of the genes UGE2 (UGE1, CNAG00697),

735 GAL1 (GAL101, CNAG_03946), and GAL7 (GAL701, CNAG_03875). Previous studies have shown

736 UGE2 is required for growth on galactose, whereas it paralogous gene UGE1 is necessary for

737 growth at $37^{\circ} \mathrm{C}$ and glucuronoxylomannogalactan (GXMGal) biosynthesis, which makes up an

738 important fraction of the Cryptococcus polysaccharide capsule (MoYRAND et al. 2008). Interestingly,

739 we previously reported that a uge $2 \Delta$ mutant strain was able to grow on galactose at $37^{\circ} \mathrm{C}$,

740 suggesting that UGE1 is able to compensate in the absence of UGE2 at $37^{\circ} \mathrm{C}$. The GAL cluster with 
741 five genes has also been lost in all other Cryptococcus species that were assessed. Thus, the $C$.

742 gattii clade species possess the only non-clustered paralogues of the GAL pathway; yet, they are

743 all able to grow on galactose as a sole carbon source, suggesting these genes are involved in both

744 GXMGal synthesis and galactose assimilation in this species (Figure S2B).

Gene ontology (GO) term enrichment analysis (PRIEBE et al. 2014) of 52 genes within 18

non-subtelomeric clusters that were absent in R265 revealed a statistically significant enrichment

of genes coding for proteins implicated in transport and transcription regulation (Figure 7A).

748 Functional annotation of these genes confirmed this result (Table S7). We identified 13 clusters

containing at least one gene coding for a putative transporter, including six MFS-type transporters, and eight clusters containing at least one gene coding for an annotated or putative transcription

751 factor (TF), including six fungal $\mathrm{Zn}(2)-\mathrm{Cys}(6)$ binuclear cluster domain-containing TFs. Overall, 752 seven clusters contain both a transporter and a TF (Figure 7B, Figure S3). Strikingly, this association 753 between transporters and TFs resembles the organization of primary metabolic gene clusters 754 (MGCs) (RoKAs et al. 2018). Because three MGCs were located within subtelomeric loci, we compared the gene content within subtelomeric regions to the gene content of the lost clusters. We considered the 20 most distal genes of each chromosome arm in H99. GO term enrichment analysis of these $560 \mathrm{H} 99$ subtelomeric genes revealed very similar profiles to the profiles obtained for the cluster genes. Again, genes coding for proteins implicated in transport in subtelomeric regions were significantly enriched (Figure 7C). 
A

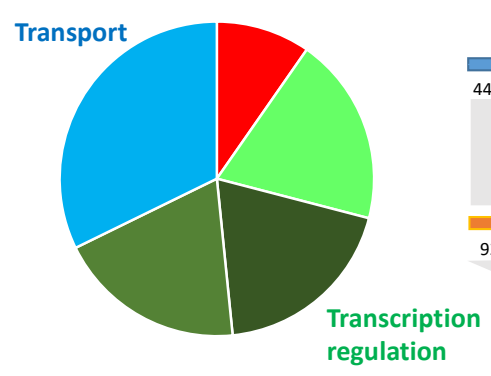

B

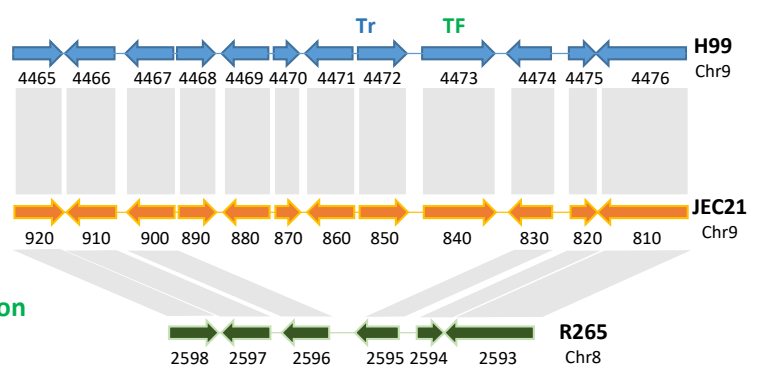

$\begin{array}{llllll}2598 & 2597 & 2596 & 25952594 & 2593 & \text { Chr8 }\end{array}$

761 Figure 7. (A) GO term enrichment analysis of genes in clusters absent in R265. Green colors

762 indicate GO terms associated with transcription regulation (GO:0006012, GO:0000981,

763 GO:0006366, GO:0006357). Blue colors indicate GO terms associated with transport

764 (GO:0055085). Orange colors indicate GO terms associated with galactose metabolism

765 (GO:0006012). (B) Example of the organization of an MGC-like cluster absent in R265.

CNAG_04468 (CNI00890) encodes a putative tartrate dehydrogenase, CNAG_04469 (CNI99880)

encodes a putative 4-aminobutyrate transaminase, CNAG_04470 (CNI00870) encodes a putative

768 halo-acid dehalogenase, CNAG_04471 (CNI00860) encodes an FAD-dependent oxidoreductase

769 superfamily protein, CNAG_04472 (CNI00850) encodes an MFS protein, and CNAG_04473

770 (CNI00840) encodes a TF with a fungal Zn(2)-Cys(6) binuclear cluster domain. (C) GO term

771 enrichment analysis of subtelomeric genes in H99. Green colors indicate GO terms associated with

772 transcription regulation (GO:0051213, GO:0000981, GO:0006366, GO:0006357, GO:0006351,

773 GO:0006355). Blue colors indicate GO terms associated with transport (GO:0055085, G0:0022891,

774 GO:0022857, GO:0005215, GO:0016021, GO:0008643, GO:0006810). The orange color indicates a

775 GO term associated with dioxygenase activity (GO:0051213). 
777 Functional annotation of these subtelomeric genes confirmed this enrichment of transporters and TFs (Table S8). We found an unexpected number of genes encoding annotated or putative TFs ( $\mathrm{n}=$ 33) and transporters $(n=68)$ within these regions of the H99 genome. Most of these TFs and transporters belong to the fungal $\mathrm{Zn}(2)$-Cys(6) binuclear cluster domain-type $(n=24)$ and MFS-type

$781(n=49)$ families, respectively. Comparison of the organization of $C$. neoformans H99 subtelomeric loci to those in $C$. deneoformans JEC21 revealed a very similar organization, and only four mosaic 783 subtelomeric regions were identified with genes from at least two different regions in H99; few 784 genes were present in H99 but absent in JEC21 (Figure 8). However, we did identify two duplicated 785 regions in the JEC21 subtelomeric regions. The first duplicated locus consists of six genes with orthologues in subtelomeric region of the left arm of Chr 5 in H99. The second duplicated region has been previously described (FRASER et al. 2005). It is located in the left arms of Chrs 8 and 12 and resulted from a telomere-telomere fusion that occurred during the construction of the JEC20/JEC21 congenic mating pair. Interestingly, a TF with a fungal Zn(2)-Cys(6) binuclear cluster domain (FZC2, CNAG_05255) and a putative amino acid transporter (CNAG_05254) are present within this repeated region. Conversely, genes in the subtelomeric regions of the right arms of H99 R265 have undergone more rearrangements compared to JEC21 - in R265 there are fifteen mosaic subtelomeric regions that contain genes from at least two different regions in H99. We also identified nine genes within six R265 subtelomeric regions whose orthologues are located far from

797 the telomeres in H99. Interestingly, functional annotation of the R265-specific subtelomeric gene 798 clusters $(n=12)$ (Figure 8; Table S9), revealed an enrichment of genes encoding TFs $(n=2)$ and transporters $(n=6)$. 
bioRxiv preprint doi: https://doi.org/10.1101/2020.09.01.278374; this version posted December 14, 2020. The copyright holder for this preprint (which was not certified by peer review) is the author/funder, who has granted bioRxiv a license to display the preprint in perpetuity. It is made available under aCC-BY-NC-ND 4.0 International license.

806 
bioRxiv preprint doi: https://doi.org/10.1101/2020.09.01.278374; this version posted December 14, 2020. The copyright holder for this preprint (which was not certified by peer review) is the author/funder, who has granted bioRxiv a license to display the preprint in perpetuity. It is made available under aCC-BY-NC-ND 4.0 International license.

\section{C. neoformans $\mathrm{H99}$}

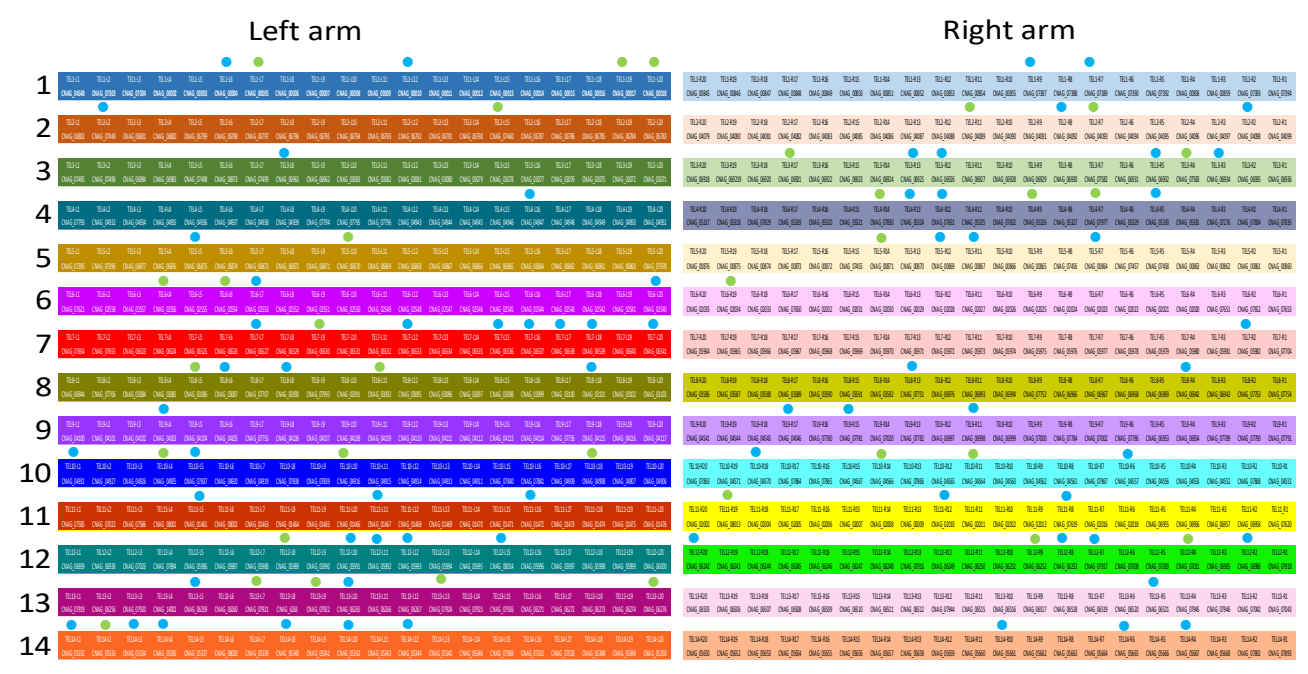

C. deneoformans JEC21

Left arm

Right arm

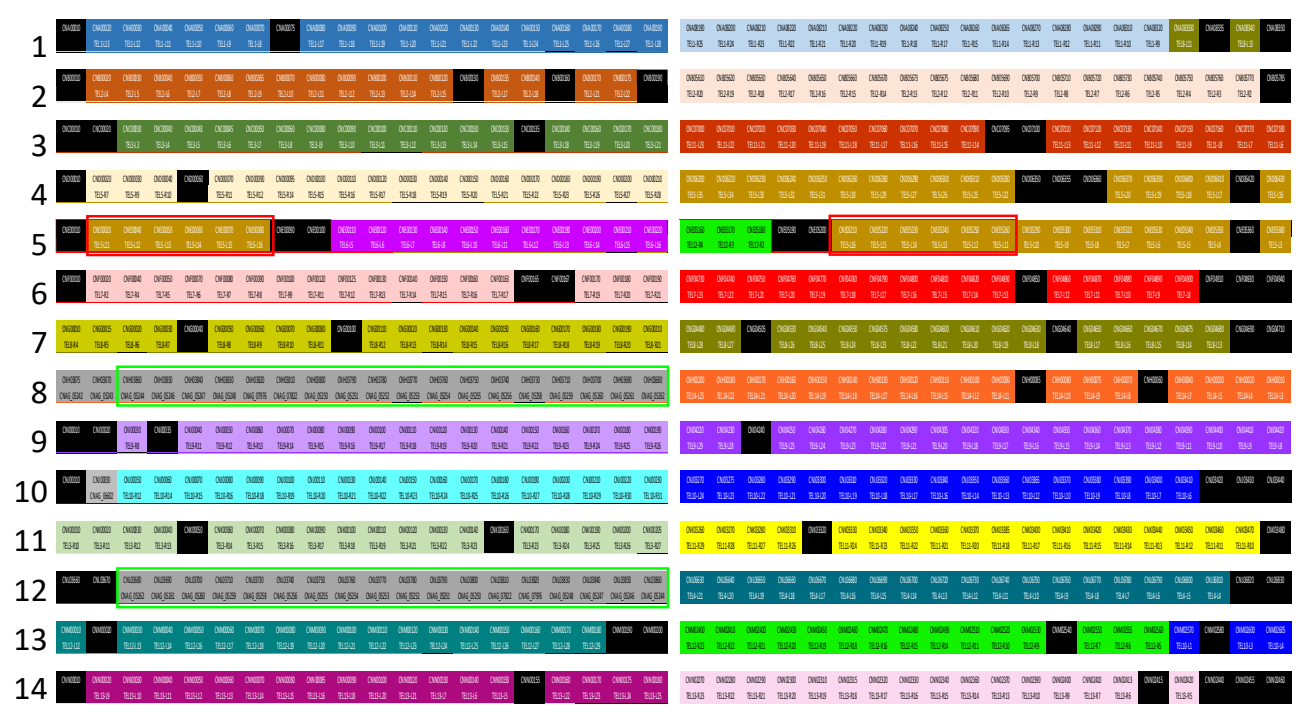

$\square \square$ duplication

C. deuterogattii R265

Left arm

Right arm

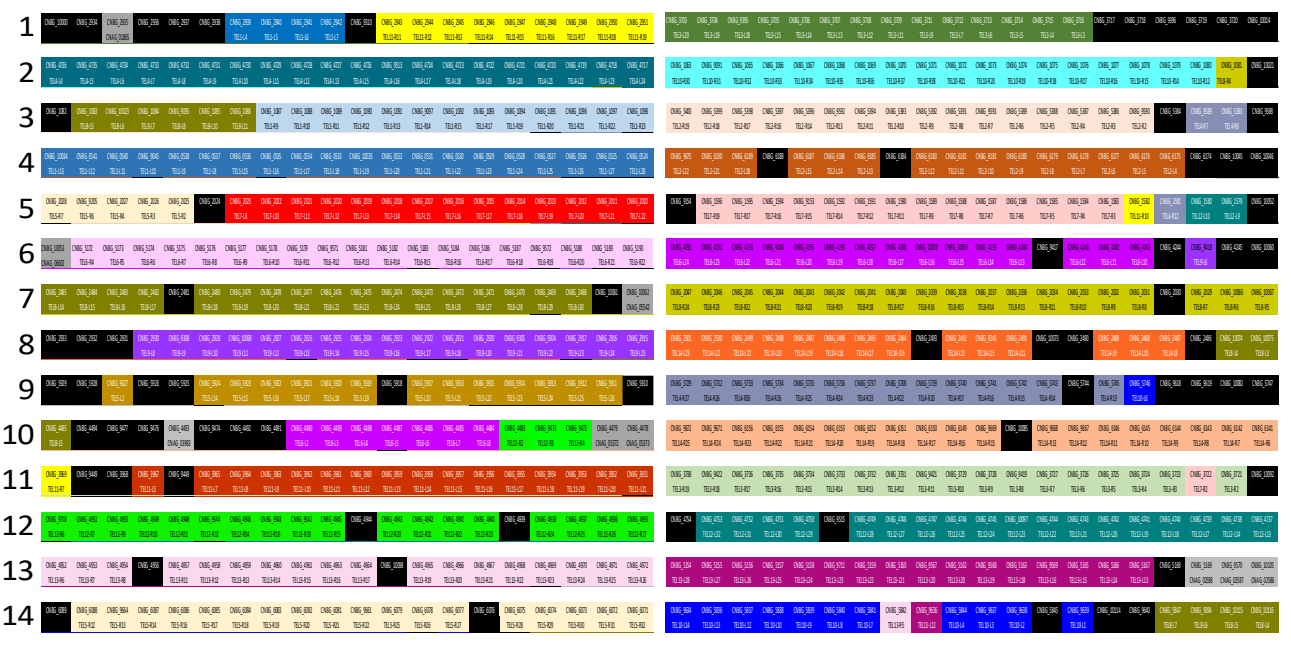


810 Figure 8. Subtelomeric gene organization in Cryptococcus. The 20 most distal genes at each

811 subtelomeric locus were considered. The color code identifies each subtelomeric regions in H99

812 and orthologous genes in the other species. The positions of these orthologues in the H99

813 subtelomeric regions are given (TEL-RX or TEL-LX correspond to genes positioned within the right

814 or left arm of chromosome $\mathrm{X}$ ). When the orthologous gene is not located within a subtelomeric

815 region, its locus named is given. Black boxes correspond to genes present in $C$. deneoformans or $C$.

816 deuterogattii but absent in C. neoformans. Red and green boxes indicate duplicated sets of genes.

817 Blue dots indicate transporters. Green dots indicate transcription factors.

819 Subtelomeric regions have been shown to be silenced by H3K27me3 histone modifications in $C$.

820 neoformans, and a large number of genes that are upregulated upon deletion of the H3K27

821 methyltransferase (encoded by EZH2) are located within subtelomeric regions (DUMESIC et al.

822 2015). Accordingly, we observed that expression of the 580 most proximal genes was generally

823 lower than the expression of the most telomere-distal genes (Figure 9). Interestingly, we found

824 that the H99 genes present within the MGCs that were lost in R265 were also poorly expressed.

825 However, none of these genes were upregulated upon EZH2 deletion (DUMESIC et al. 2015),

826 suggesting that they are not directly regulated by H3K7me3. In summary, these data suggest that

827 dynamic exchanges of MGCs between subtelomeric regions occurred during Cryptococcus

828 speciation. These results also suggest that MGC exchanges between subtelomeric loci and more

829 central parts of chromosomes might be associated with new assimilation capacities. 


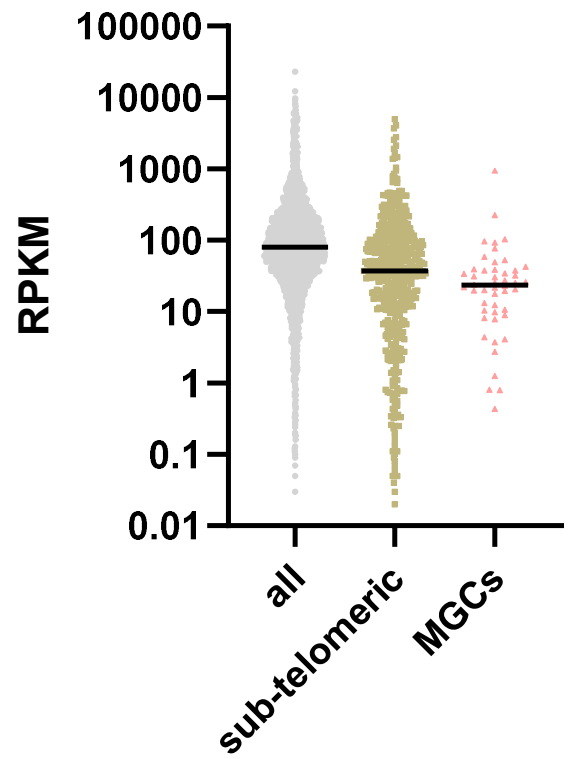

832 Figure 9. Expression of genes according to their position on the chromosome. Subtelomeric genes

833 are the 20 most distal genes of each chromosome arm. The $\mathrm{H} 99$ genes present within the non-

834 subtelomeric cluster of genes lost in R265 are indicated as MGCs.

835

836 
bioRxiv preprint doi: https://doi.org/10.1101/2020.09.01 278374 this version posted December 14,2020 . The copyright holder for this preprint (which was not certified by peer review) is the author/funder, who has granted bioRxiv a license to display the preprint in perpetuity. It is made available under aCC-BY-NC-ND 4.0 International license.

\section{Discussion}

Although a number of bioinformatic pipelines have been published in recent years, accurate annotation of fungal genomes is still difficult due to their complexity and compactness (HAAS et al. 2011). In this study, we have carefully optimized a previously published CufflinksCodingQuarry-based annotation pipeline and tested it on two complex fungal genomes. This pipeline largely outcompeted the BRAKER1 pipeline when applied to two Cryptococcus reference genomes (C. neoformans H99 and C. deneoformans JEC21) and would likely outcompete many other pipelines use to annotate fungal genomes de novo (MIN et al. 2017).

Our optimization process revealed three notable points. First and counterintuitively, increasing the quantity of data did not always result in a better annotation. This is likely because Cufflinks tends to make huge clusters when large data sets are input; these clusters might be eliminated during the transcript identification step. Accordingly, we found that the number of predicted transcripts decreased when too much data was used. Second, we found a nearly linear relationship between the number of introns predicted and the quality of the annotation. However, this correlation did not hold when two of the pipelines were compared; the BRAKER pipeline predicted more introns than the $\mathrm{C} 3 \mathrm{Q}$ pipeline, along with predicting many more genes.

Nevertheless, the correlation between intron number and annotation quality provided a facile way to evaluate the reliability of a de novo annotation, which might be affected, for instance, by the quality of the RNA-seq data. Third, we found the final step of comparative genomics did not always improve the quality of the annotation. In our assay, the Exonerate-based analysis step using the whole proteome of a reference species primarily introduced errors into the annotation.

This was probably due to the fact that even when manually curating genome annotations, a number of dubious genes remain, which are then transferred to the new genome annotation. In fact, a systematic usage of a comparative annotation step following a de novo RNA-seq annotation 
861 would likely result in a dramatic expansion of dubiously annotated genes in fungal genomes.

862 Accordingly, it is noticeable that the number of predicted coding genes in R265 $(n=6405)$ is lower

863 than the ones predicted in H99 $(n=6795)$ and JEC21 ( $n=6639)$ although we ignore whether these

864 differences have some biological relevance or if they are due to the different strategies used to

865 annotate these genomes.

866

During the annotation of the R265 genome, we manually curated a subset of genes that were lost in R265 compared to all of the other Cryptococcus species as well as a set of putative pseudogenes. The identification of genes specifically lost or pseudogenized in R265 has previously been used as a strategy to identify novel RNAi components in C. neoformans (FERETZAKI et al. 2016). Accordingly, most known RNAi genes are present in these sets of lost and pseudogenized genes (BILLMYRE et al. 2013). However, some genes, like RDE1 (BURKE et al. 2019), which is necessary for siRNA production, are present and functional in R265, suggesting that it may have roles independent from RNAi silencing. On the other hand, GWO1, which is also considered to be an RNAi pathway component, is also absent in the $C$. tetragattii strain IND107 and is therefore absent in our list as well. One possible explanation is that Gwo1 alone or in complex with Ago1 could play another role independent of RNAi. Another possibility is that a Gwo1-dependent RNAi pathway has also been lost in C. tetragattii. Nevertheless, this analysis confirms that looking for specific gene loss in a fungal species deficient for a certain pathway remains a promising strategy for the identification of genes implicated in this pathway in other proficient species. In the present case, it would be interesting to see how many of the R265 truncated genes are functional in other $C$. gattii species, although it would demand a complex manual curation, which is beyond the scope of this paper.

Our study revealed that loss of RNAi in R265 is associated with few general transcriptome modifications compared to the transcriptomes of JEC21 and H99, aside from the predictable 
absence of siRNA. This might be because we did not annotate most non-coding features like IncRNAs, transcript leaders, and 3'UTRs. Yet, quantification of the sense/antisense transcription

ratio at CDS did not reveal any differences between R265 and the other Cryptococcus species analyzed, suggesting that this ratio does not depend on the RNAi status of the species in this genus. This is in agreement with the fact that siRNAs in C. neoformans primarily target transposons and retrotransposons (JANBON et al. 2010; WANG et al. 2010; DUMESIC et al. 2013), whereas antisense transcription is associated with nearly all of the genes as we have shown. This result also suggests antisense transcription in Cryptococcus only rarely results in the production of doublestranded RNA. Dumesic and colleagues showed that delayed splicing is a source of siRNA production in C. neoformans (DUMESIC et al. 2013). We thus anticipated that the absence of RNAi would increase the level of intron retention. In agreement with previous reports in $C$. deneoformans, we found that IR level was regulated by growth conditions in both $C$. neoformans and C. deuterogattii (GONZALEZ-HILARION et al. 2016). However, the number of introns regulated by IR was markedly larger in R265 suggesting that IR is better tolerated in this RNAi-deficient species. We also expected that some compensatory mechanisms might be acting to control the level of IR because IR rates were largely similar across the three species analyzed even though it was higher 901 in $\mathrm{R} 265$, at least at $37^{\circ} \mathrm{C}$. It is important to note the remarkable effect of temperature on both IR and antisense transcription, which might be related to a recent report that transposons are specifically mobilized at this temperature in C. deneoformans (GUSA et al. 2020). contain only a single gene, we also identified gene clusters that were missing in R265. Analysis of

906 the gene content within these clusters revealed a strong enrichment of genes coding for proteins 907 implicated in transport and transcriptional regulation. This finding was reminiscent of patterns 908 identified in metabolic gene clusters (MGCs) involved in primary metabolism, which typically 909 contain transcription factors and transporters (RoKAS et al. 2018). MGCs can be lost or gained in 46 
910 fungi and several examples of instances of horizontal transfer of whole MGCs from one species to

911 another have been published (SLOT AND ROKAS 2010; ROKAS et al. 2018; WANG et al. 2019). In

912 filamentous fungi, the majority of MGCs are located within subtelomeric regions, which are largely

913 subjected to inter-chromosomal reshuffling (GLADIEUX et al. 2014). Two examples of lineage-

914 specific gene clusters harboring both transcription factors and transporters have been previously

915 reported in C. neoformans (RHODES et al. 2017), suggesting dynamic gene cluster gain and loss

916 events even with a single species in Cryptococcus. Interestingly, these $C$. neoformans lineage-

917 specific clusters also contain transcription factors and transporters (RHODES et al. 2017). A more

918 recent report suggests that genes within one of these $C$. neoformans clusters are co-regulated, as

919 is expected from a typical MGC (Yu et al. 2020). In Cryptococcus, we found that the subtelomeric

920 regions were also enriched for characteristic MGC genes as well, and comparisons of subtelomeric

921 gene organization across the three Cryptococcus species suggested active reshuffling. This was in

922 agreement with previous data showing that subtelomeric genes are under strong evolutionary

923 pressure in Cryptococcus (DESJARDINS et al. 2017). We found a large number of genes encoding

924 transporters and TFs of unknown function in Cryptococcus subtelomeric regions. Surprisingly, most

925 of the TF genes identified in these MGCs within subtelomeric regions as well as in MGCs far from

926 telomeres were not annotated as TFs and were not included when a systematic TF deletion

927 collection was constructed and studied (JUNG et al. 2015). It therefore seems that the TF repertoire

928 in Cryptococcus may be larger than currently appreciated. Similarly, besides myo-inositol

929 transporters, which have been previously reported to be localized within subtelomeric regions

930 (XUE et al. 2010), the substrates of most transporters located in these regions remain unknown.

931 Genes within subtelomeric regions are silenced by H3K27me3 epigenetic marks and,

932 accordingly, are expressed at lower levels than genes located in more central regions of the

933 chromosomes. Similarly, genes within the subtelomeric clusters lost in R265 were poorly

934 expressed. Yet, their expression levels did not significantly change following deletion of the gene 
935 encoding the $\mathrm{H} 3 \mathrm{~K} 27$ me3 methyltransferase $E Z H 2$, suggesting they are either not regulated by

936 H3K27me3 or that additional changes are needed to activate expression of these genes like those

937 previously described in Fusarium graminearum (Connolly et al. 2013). If this is the case, the

938 regulation of GAL genes by galactose might represent a good example of how genes within the

939 MGCs could be regulated in Cryptococcus (WICKES AND EdMAN 1995; MoYRAND et al. 2008; Ruff et al.

940 2009). Besides the GAL cluster, the function and regulation of most of MGC genes in Cryptococcus

941 are unknown. Nevertheless, our results suggest active exchange between subtelomeric regions

942 and more central parts of chromosomes in Cryptococcus, potentially reshaping primary

943 metabolism for adaptation to different environmental niches. They also emphasize how both

944 complete genome and precise annotations are needed to study these dynamics in fungi. 
Acknowledgements

PAGF was supported a CAPES exchange grant (Advanced Network of Computational Biology

948 - RABICÓ - Grant no. 23038.010041/2013-13). This work was supported by a CAPES COFECUB grant $949 \mathrm{n}^{\circ} 39712 Z \mathrm{~K}$ to GJ, by a CNPq grant (309897/2017-3) to CCS, and by a NIH/NIAID R37 MERIT Award 950 AI39115-23 and a NIH/NIAID R01 Award AI50113-16 to J.H. J.H. is co-director and fellow of CIFAR 951 program Fungal Kingdom: Threats \& Opportunities. S.J.P was supported by the NIH/NIAID F31 952 Fellowship 1F31Al143136-02. Members of the Heitman laboratory are acknowledged for valuable 953 discussion. 
bioRxiv preprint doi: https://doi.org/10.1101/2020.09.01.278374; this version posted December 14,2020 . The copyright holder for this preprint (which was not certified by peer review) is the author/funder, who has granted bioRxiv a license to display the preprint in perpetuity. It is made available under aCC-BY-NC-ND 4.0 International license.

957

958 
References

Alcid, E. A. and T. Tsukiyama, 2016 Expansion of antisense IncRNA transcriptomes in budding yeast species since the loss of RNAi. Nature Structural and Molecular Biology 23: 450-455.

Anders, S., P. T. Pyl and W. Huber, 2014 HTSeq-a Python framework to work with high-throughput sequencing data. Bioinformatics 31: 166-169.

Basenko, E. Y., J. A. Pulman, A. Shanmugasundram, O. S. Harb, K. Crouch et al., 2018 FungiDB: an integrated bioinformatic resource for fungi and oomycetes. Journal of Fungi (Basel, Switzerland) 4: 39.

Billmyre, R. B., S. Calo, M. Feretzaki, X. Wang and J. Heitman, 2013 RNAi function, diversity, and loss in the fungal kingdom. Chromosome Research 21: 561-572.

Burke, J. E., A. D. Longhurst, P. Natarajan, B. Rao, J. Liu et al., 2019 A non-Dicer RNase III and four other novel factors required for RNAi-mediated transposon suppression in the human pathogenic yeast Cryptococcus neoformans. G3 (Bethesda, Md.) 9: 2235-2244.

Cantarel, B. L., I. Korf, S. M. C. Robb, G. Parra, E. Ross et al., 2008 MAKER: an easy-to-use annotation pipeline designed for emerging model organism genomes. Genome Research 18: 188-196. 
Cheng, P.-Y., A. Sham and J. W. Kronstad, 2009 Cryptococcus gattii isolates from the British Columbia cryptococcosis outbreak induce less protective inflammation in a murine model of infection than Cryptococcus neoformans. Infection and Immunity 77: 4284.

Conesa, A., S. Götz, J. M. García-Gómez, J. Terol, M. Talón et al., 2005 Blast2GO: a universal tool for annotation, visualization and analysis in functional genomics research. Bioinformatics 21: 3674-3676.

Connolly, L. R., K. M. Smith and M. Freitag, 2013 The Fusarium graminearum histone H3 K27 methyltransferase KMT6 regulates development and expression of secondary metabolite gene clusters. PLOS Genetics 9: e1003916.

D'Souza, C. A., J. W. Kronstad, G. Taylor, R. Warren, M. Yuen et al., 2011 Genome variation in Cryptococcus gattii, an emerging pathogen of immunocompetent hosts. mBio 2: e0034200310.

Dal Molin, A., A. Minio, F. Griggio, M. Delledonne, A. Infantino et al., 2018 The genome assembly of the fungal pathogen Pyrenochaeta lycopersici from Single-Molecule Real-Time sequencing sheds new light on its biological complexity. PLOS One 13: e0200217.

Desjardins, C. A., C. Giamberardino, S. M. Sykes, C.-H. Yu, J. L. Tenor et al., 2017 Population genomics and the evolution of virulence in the fungal pathogen Cryptococcus neoformans. Genome research 27: 1207-1219.

Ding, C., Yin, J., Tovar, E.M.M., Fitzpatrick, D.A., Higgins, D.G. and Thiele, D.J. (2011) The copper regulon of the human fungal pathogen Cryptococcus neoformans H99. Mol Microbiol, 81, 1560-1576.

Dumesic, Phillip A., Christina M. Homer, James J. Moresco, Lindsey R. Pack, Erin K. Shanle et al., 2015 Product binding enforces the genomic specificity of a yeast polycomb repressive complex. Cell 160: 204-218. 
Dumesic, P. A., P. Natarajan, C. Chen, I. A. Drinnenberg, B. J. Schiller et al., 2013 Stalled spliceosomes are a signal for RNAi-mediated genome defense. Cell 152: 957-968.

Dunn, N. A., D. R. Unni, C. Diesh, M. Munoz-Torres, N. L. Harris et al., 2019 Apollo: Democratizing genome annotation. PLOS Computational Biology 15: e1006790.

Emms, D. M., and S. Kelly, 2019 OrthoFinder: phylogenetic orthology inference for comparative genomics. Genome Biology 20: 238.

Farrer, R. A., C. A. Desjardins, S. Sakthikumar, S. Gujja, S. Saif et al., 2015 Genome evolution and innovation across the four major lineages of Cryptococcus gattii. mBio 6: e00868-00815. gattii (VGV) discovered in the Central Zambezian Miombo Woodlands. mBio 10:e02306-19.

Ferrareze, P. A. G., R. S. A. Streit, P. R. dos Santos, F. M. dos Santos, R. M. C. de Almeida et al., 2017 Transcriptional analysis allows genome reannotation and reveals that Cryptococcus gattii polymorphism illuminates loss and retention of novel RNAi silencing components in the Cryptococcus pathogenic species complex. PLOS Genetics 12: e1005868-e1005868.

Giordano, F., L. Aigrain, M. A. Quail, P. Coupland, J. K. Bonfield et al., 2017 De novo yeast genome assemblies from MinION, PacBio and MiSeq platforms. Scientific Reports 7: 3935. provides insight into the mechanisms of adaptive divergence in eukaryotes. Molecular Ecology 23: 753-773. 
1030 Gonzalez-Hilarion, S., D. Paulet, K.-T. Lee, C.-C. Hon, P. Lechat et al., 2016 Intron retentiondependent gene regulation in Cryptococcus neoformans. Scientific Reports 6: 32252.

1032 Grabherr, M. G., B. J. Haas, M. Yassour, J. Z. Levin, D. A. Thompson et al., 2011 Full-length transcriptome assembly from RNA-seq data without a reference genome. Nature

Gusa, A., J. D. Williams, J.-E. Cho, A. F. Averette, S. Sun et al., 2020 Transposon mobilization in the human fungal pathogen Cryptococcus is mutagenic during infection and promotes drug resistance in vitro. Proceedings of the National Academy of Sciences of the USA 117: 9973.

Haas, B. J., Q. Zeng, M. D. Pearson, C. A. Cuomo and J. R. Wortman, 2011 Approaches to fungal genome annotation. Mycology 2: 118-141.

Hagen, F., K. Khayhan, B. Theelen, A. Kolecka, I. Polacheck et al., 2015 Recognition of seven species in the Cryptococcus gattii/Cryptococcus neoformans species complex. Fungal Genetics and

Haridas, S., A. Salamov and I. V. Grigoriev, 2018 Fungal Genome Annotation, pp. 171-184 in Fungal Genomics: Methods and Protocols, edited by R. P. de Vries, A. Tsang and I. V. Grigoriev.

Hoff, K. J., S. Lange, A. Lomsadze, M. Borodovsky and M. Stanke, 2016 BRAKER1: unsupervised RNAseq-based genome annotation with GeneMark-ET and AUGUSTUS. Bioinformatics (Oxford, England) 32: 767-769.

Huang, M., Hebert, A.S., Coon, J.J. and Hull, C.M. (2015) Protein composition of infectious spores

Huston, S. M., S. S. Li, D. Stack, M. Timm-McCann, G. J. Jones et al., 2013 Cryptococcus gattii is killed reveals novel sexual development and germination factors in Cryptococcus. PLoS Genet, 11, by dendritic cells, but evades adaptive immunity by failing to induce dendritic cell maturation. The Journal of Immunology 191: 249. 
1055 James, T. Y., J. E. Stajich, C. T. Hittinger and A. Rokas, 2020 Toward a fully resolved fungal tree of life. Annual Review of Microbiology 74.

Janbon, G., 2018 Introns in Cryptococcus. Memorias do Instituto Oswaldo Cruz 113: e170519e170519.

1059 Janbon, G., S. Y. Maeng, D-F., Y.-J. Ko, K.-W. Jung, F. Moyrand et al., 2010 Characterizing the role of RNA silencing components in Cryptococcus neoformans. Fungal Genetics and Biology 47: 1070-1080.

Janbon, G., K. L. Ormerod, D. Paulet, E. J. Byrnes III, G. Chatterjee et al., 2014 Analysis of the genome and transcriptome of Cryptococcus neoformans var. grubii reveals complex RNA expression and microevolution leading to virulence attenuation. PLOS Genetics 10: e1004261.

Jung, K.-W., D.-H. Yang, S. Maeng, K.-T. Lee, Y.-S. So et al., 2015 Systematic functional profiling of transcription factor networks in Cryptococcus neoformans. Nature Communications 6: 6757.

Kidd, S. E., F. Hagen, R. L. Tscharke, M. Huynh, K. H. Bartlett et al., 2004 A rare genotype of Cryptococcus gattii caused the cryptococcosis outbreak on Vancouver Island (British Columbia, Canada). Proceeding of the National Academy of Sciences of the USA 101: 1725817263.

1071 Kim, D., G. Pertea, C. Trapnell, H. Pimentel, R. Kelley et al., 2013 TopHat2: accurate alignment of transcriptomes in the presence of insertions, deletions and gene fusions. Genome biology 14: R36-R36. Harbor Perspectives in Medicine 4: a019760.

1077 Lam, W. C., R. Upadhya, C. A. Specht, A. E. Ragsdale, C. R. Hole et al., 2019 Chitosan biosynthesis and virulence in the human fungal pathogen Cryptococcus gattii. mSphere 4: e00644-00619. 
1079 Langmead, B., and S. L. Salzberg, 2012 Fast gapped-read alignment with Bowtie 2. Nature Methods 9: 357-359.

1081 Lee, K.-T., So, Y.-S., Yang, D.-H., Jung, K.-W., Choi, J., et al. (2016) Systematic functional analysis of kinases in the fungal pathogen Cryptococcus neoformans. Nature Communications, 7, 12766.

Lomsadze, A., P. D. Burns and M. Borodovsky, 2014 Integration of mapped RNA-seq reads into automatic training of eukaryotic gene finding algorithm. Nucleic Acids Research 42: e119-

Lowe, T. M., and P. P. Chan, 2016 tRNAscan-SE On-line: integrating search and context for analysis of transfer RNA genes. Nucleic Acids Research 44: W54-W57.

Ma, H., F. Hagen, D. J. Stekel, S. A. Johnston, E. Sionov et al., 2009 The fatal fungal outbreak on Vancouver Island in characterized by enhanced intracellular parasitism driven by mitochondrial regulation. Proceeding of the National Academy of Sciences of the USA 106:

Martin, M., 2011 Cutadapt removes adapter sequences from high-throughput sequencing reads. EMBnet.journal; Vol 17, No 1: Next Generation Sequencing Data Analysis.

Min, B., I. V. Grigoriev and I.-G. Choi, 2017 FunGAP: Fungal Genome Annotation Pipeline using evidence-based gene model evaluation. Bioinformatics 33: 2936-2937.

Moyrand, F., I. Lafontaine, T. Fontaine and G. Janbon, 2008 UGE1 and UGE2 regulate the UDPglucose/UDP-galactose equilibrium in Cryptococcus neoformans. Eukaryotic Cell 7: 2069-

Mudge, J. M., and J. Harrow, 2016 The state of play in higher eukaryote gene annotation. Nature Reviews Genetics 17: 758-772.

1101 Ngamskulrungroj, P., Y. Chang, E. Sionov and K. J. Kwon-Chung, 2012 The primary target organ of 
1104 Pertea, G., and M. Pertea, 2020 GFF Utilities: GffRead and GffCompare. F1000Research 9: 304.

1105 Priebe, S., C. Kreisel, F. Horn, R. Guthke and J. Linde, 2014 FungiFun2: a comprehensive online resource for systematic analysis of gene lists from fungal species. Bioinformatics 31: 445-

Rhodes, J., C. A. Desjardins, S. M. Sykes, M. A. Beale, M. Vanhove et al., 2017 Tracing genetic exchange and biogeography of Cryptococcus neoformans var. grubii at the global population level. Genetics: 10.1534/genetics.1117.203836.

Rokas, A., J. H. Wisecaver and A. L. Lind, 2018 The birth, evolution and death of metabolic gene clusters in fungi. Nature Reviews Microbiology 16: 731-744.

Ruff, J. A., J. K. Lodge and L. G. Baker, 2009 Three galactose inducible promoters for use in $C$. neoformans var. grubii. Fungal Genetics and Biology 46: 9-16.

Semighini C.P., A.F. Averette, J.R. Perfect, and J. Heitman, 2011. Deletion of Cryptococcus neoformans AIF ortholog promotes chromosome aneuploidy and fluconazole-resistance in a metacaspase-independent manner. PLOS Pathogens 7: e1002364.

Sharpe, D. 2015 Chi-Square Test is Statistically Significant: Now What?, Practical Assessment. Research, and Evaluation: Vol. 20 , Article 8. DOI: https://doi.org/10.7275/tbfa-x148.

Shen, X.-X., D. A. Opulente, J. Kominek, X. Zhou, J. L. Steenwyk et al., 2018 Tempo and mode of genome evolution in the budding yeast subphylum. Cell 175: 1533-1545.e1520.

Slater, G. S., and E. Birney, 2005 Automated generation of heuristics for biological sequence comparison. BMC Bioinformatics: 31.

Slot, J. C., and A. Rokas, 2010 Multiple GAL pathway gene clusters evolved independently and by different mechanisms in fungi. Proceeding of the National Academy of Sciences of the USA 107: 10136-10141.

1127 Stanke, M., M. Diekhans, R. Baertsch and D. Haussler, 2008 Using native and syntenically mapped 
1129 Testa, A. C., J. K. Hane, S. R. Ellwood and R. P. Oliver, 2015 CodingQuarry: highly accurate hidden Markov model gene prediction in fungal genomes using RNA-seq transcripts. BMC Genomics 16: 170.

1132 Törönen, P., A. Medlar and L. Holm, 2018 PANNZER2: a rapid functional annotation web server. Nucleic Acids Research 46: W84-W88.

1134 Trapnell, C., B. A. Williams, G. Pertea, A. Mortazavi, G. Kwan et al., 2010 Transcript assembly and quantification by RNA-seq reveals unannotated transcripts and isoform switching during cell differentiation. Nature Biotechnology 28: 511-515.

1138 Wallace, E. W. J., C. Maufrais, J. Sales-Lee, L. R. Tuck, L. de Oliveira et al., 2020 Quantitative global studies reveal differential translational control by start codon context across the fungal kingdom. Nucleic Acids Research 48: 2312-2331.

Wang, M., H. Fu and R. Ruan, 2019 A small horizontally transferred gene cluster contributes to the sporulation of Alternaria alternata. Genome Biology and Evolution 11: 3436-3444.

1143 Wang, X., Y. P. Hsueh, W. Li, A. Floyd, R. Skalsky et al., 2010 Sex-induced silencing defends the genome of Cryptococcus neoformans via RNAi. Genes \& Development 24: 2566-2582.

Wickes, B. L., and J. C. Edman, 1995 The Cryptococcus neoformans GAL7 gene and its use as an inducible promoter. Molecular Microbiology 16: 1099-1109.

1147 Wilm, A., D. G. Higgins, F. Valentin, G. Blackshields, H. McWilliam et al., 2007 Clustal W and Clustal X version 2.0. Bioinformatics 23: 2947-2948.

1149 Xue, C., T. Liu, L. Chen, W. Li, I. Liu et al., 2010 Role of an expanded inositol transporter repertoire in Cryptococcus neoformans sexual reproduction and virulence. mBio 1: e00084-00010.

1151 Yadav, V., S. Sun, R. B. Billmyre, B. C. Thimmappa, T. Shea et al., 2018 RNAi is a critical determinant of centromere evolution in closely related fungi. Proceeding of the National Academy of 
1154 Yu, C.-H., Y. Chen, C. A. Desjardins, J. L. Tenor, D. L. Toffaletti et al., 2020 Landscape of gene biologically relevant stresses. Microbial Genomics 6.

1157 Zhu, P., B. Zhai, X. Lin, and A. Idnurm, 2013. Congenic strains for genetic analysis of virulence traits in Cryptococcus gattii. Infection and Immunity 81: 2616-2625.

1159

1160 